\title{
Red Nucleus Neurons Actively Contribute to the Acquisition of Classically Conditioned Eyelid Responses in Rabbits
}

\author{
Renny Pacheco-Calderón, Alejandro Carretero-Guillén, José M. Delgado-García, and Agnès Gruart \\ Division of Neurosciences, Pablo de Olavide University, Seville-41013, Spain
}

The red nucleus (RN) is a midbrain premotor center that has been suggested as being involved in the acquisition and/or performance of classically conditioned nictitating membrane/eyelid responses. We recorded in rabbits the activity of RN and pararubral neurons during classical eyeblink conditioning using a delay paradigm. Neurons were identified by their antidromic activation from contralateral facial and accessory abducens nuclei and by their synaptic activation from the ipsilateral motor cortex (MC) and the contralateral cerebellar interpositus (IP) nucleus. For conditioning, we used a tone as a conditioned stimulus (CS) followed $250 \mathrm{~ms}$ later by a $100 \mathrm{~ms}$ air puff as an unconditioned stimulus (US) coterminating with it. Conditioned responses (CRs) were determined from the evoked changes in the electromyographic activity of the orbicularis oculi (00) muscle. Recorded neurons were classified by their antidromic activation and by their changes in firing rate during the CS-US interval. Identified neurons increased their firing rates in relation to the successive conditioning sessions, but their discharge rates were related more to the EMG activity of the 00 muscle than to the learning curves. Reversible inactivation of the IP nucleus with lidocaine during conditioning evoked a complete disappearance of both conditioned and unconditioned eyelid responses, and a progressive decrease in CR-related activity of RN neurons. In contrast, MC inactivation evoked a decrease in the acquisition process and an initial disfacilitation of neuronal firing (which was later recovered), together with the late appearance of CRs. Thus, RN neurons presented learning-dependent changes in activity following MC inactivation.

\section{Introduction}

The $\mathrm{RN}$ is a mesencephalic premotor center related to the generation and control of locomotion and of other more specific motor behaviors, including facial motor responses. The red nucleus $(\mathrm{RN})$ receives substantial inputs from both the sensorimotor cortex and cerebellar nuclei (Miller and Gibson, 2009; Gruber and Gould, 2010). In this regard, the RN participates in the neuronal network connecting the cerebellar interpositus (IP) nucleus and the facial and accessory abducens nuclei, a neuronal circuit assumed to be involved in the acquisition and storage of classical eyeblink conditioning (Krupa et al., 1993). In this circuit, the RN is considered a mere relay center, while the learning-dependent changes in synaptic strength underlying the learning process take place in the IP nucleus, or in the overlying cerebellar cortex (Bracha et al., 2009; Freeman and Steinmetz, 2011). These contentions have been supported mainly in transient or permanent lesion studies (Chapman et al., 1990; Clark and Lavond, 1993), because there are only a few studies that have recorded and analyzed the firing activities of putative $\mathrm{RN}$ neurons during the acquisition and performance of classically conditioned nictitating

Received April 12, 2012; revised July 6, 2012; accepted July 14, 2012.

Author contributions: J.M.D.-G. and A.G. designed research; R.P.-C., A.C.-G., J.M.D.-G., and A.G. performed research; R.P.-C., A.C.-G., J.M.D.-G., and A.G. analyzed data; R.P.-C., J.M.D.-G., and A.G. wrote the paper.

This study was supported by grants from the Spanish MINECO (BFU2011-29089 and BFU2011-29286) and Junta de Andalucía (BI0122, CVI 2487, and P07-CVI-02686). R.P.-C. is a visiting researcher from the Department of Biology,

Faculty of Science and Technology, University of Carabobo, Venezuela. We thank Leopoldo Pérez-Rosendo for animal handling and care and Roger Churchill for his help in manuscript editing.

Correspondence should be addressed to Professor Agnès Gruart, División de Neurociencias, Universidad Pablo de Olavide, Ctra. de Utrera, Km. 1, 41013-Sevilla, Spain. E-mail: agrumas@upo.es.

DOI:10.1523/JNEUROSCI.1782-12.2012

Copyright $\odot 2012$ the authors $\quad 0270-6474 / 12 / 3212129-15 \$ 15.00 / 0$ membrane (Desmond and Moore, 1991) or eyelid (PorrasGarcía et al., 2010) responses.

It was initially assumed that rubral projections to the facial muscles were collateral to descending rubrospinal axons originated at the magnocellular division of the RN (Courville, 1966). However, the use of more specific neuroanatomical tracing techniques has disproved such a contention, confirming that rubral projections to the facial and the accessory abducens nucleus originate in a specific dorsolateral subdivision of the parvocellular $\mathrm{RN}$, including the pararubral area (Ruigrok and Cella, 1995; Morcuende et al., 2002). This information imposes the need for the proper identification of recorded RN units to precisely determine their contribution to the learning process. In addition, the RN appears to be a junction center (Pong et al., 2008) under the hierarchical control of both the cerebral and cerebellar cortices with a more than passive role in motor control. The selective transient inactivation of these two RN afferent pathways could give a more complete picture of the specific roles of RN neurons in associative learning.

To settle these questions, rabbits were prepared for the chronic recording of the EMG activity of the orbicularis oculi $(\mathrm{OO})$ muscle and of the unitary activity of antidromic- and synaptically identified right RN and pararubral neurons during classical eyeblink conditioning using a delay paradigm. For conditioning, we used a $350 \mathrm{~ms}$ tone as a conditioned stimulus (CS) followed by $250 \mathrm{~ms}$ from its beginning by a $100 \mathrm{~ms}$ air puff directed at the contralateral cornea. Appropriate sites of the ipsilateral motor cortex (MC) and contralateral IP nucleus were subjected to electrical microstimulation and/or microinfusion of lidocaine. Collected results suggest that RN and pararubral neu- 
rons are involved more in the proper performance of eyelid conditioned responses (CRs) than in the acquisition process. In addition, the reversible inactivation of the MC and of the IP nucleus produced differential effects on conditioned and/or unconditioned eyelid responses. Interestingly, $\mathrm{RN}$ and pararubral neurons seemed to partially substitute the learning deficits evoked by MC, but not by IP nucleus, inactivation.

\section{Materials and Methods}

Experimental subjects. Experimental procedures were performed on adult male rabbits (New Zealand White albino; Isoquimen) weighing 2.3-3.1 $\mathrm{kg}$ on arrival. Before and after surgery, animals were maintained in the same room, but placed in independent cages. Animals were kept on a $12 \mathrm{~h}$ light/dark cycle, with a continuous control of humidity $(55 \pm 5 \%)$ and temperature $\left(21 \pm 1^{\circ} \mathrm{C}\right)$. Experiments were performed in accordance with European Union guidelines (2003/65/CE) and following Spanish regulations (RD 1201/2005) for the use of laboratory animals in chronic electrophysiological and behavioral studies. Experiments were also approved by the local Ethics Committee of the Pablo de Olavide University (Seville, Spain).

Surgical procedures. Animals were anesthetized with a ketamine-xylazine mixture (Ketaminol, $50 \mathrm{mg} / \mathrm{ml}$; Rompun, $20 \mathrm{mg} / \mathrm{ml}$; and atropine sulfate, $0.5 \mathrm{mg} / \mathrm{kg}$ ) at an initial dosage of $0.85 \mathrm{ml} / \mathrm{kg}$. Anesthesia was maintained by intravenous perfusion at a flow rate of $10 \mathrm{mg} / \mathrm{kg} / \mathrm{h}$.

As illustrated in Figures $1 A$ and $2 A$, animals were prepared for the chronic recording of unitary activity in the $\mathrm{RN}$ area during classical eyeblink conditioning. For this, under aseptic conditions, a window (6 $\mathrm{mm} \times 6 \mathrm{~mm}$ ) was drilled through the occipital bone centered on the right $\mathrm{RN}$ [anteroposterior $(\mathrm{AP})=-8.5 \mathrm{~mm}$; lateral $(\mathrm{L})=1 \mathrm{~mm}$; Girgis and Shih-Chang, 1981]. The dura mater was removed and the cortical surface was protected with an inert plastic cover. A recording chamber was built with acrylic cement around the window. The recording chamber was covered with sterile gauze and a plastic cap during nonrecording sessions. A silver electrode ( $1 \mathrm{~mm}$ in diameter) in contact with the dura mater was attached to the left parietal bone ( $A P=-10 \mathrm{~mm}, \mathrm{~L}=6 \mathrm{~mm}$ ) as a ground for local field potential recordings. Animals were also implanted with stimulating electrodes aimed at the contralateral facial $(\mathrm{AP}=-18 \mathrm{~mm}, \mathrm{~L}=3.5 \mathrm{~mm}$; dorsal $(\mathrm{D})=10 \mathrm{~mm}$ from cerebellum surface) or at the accessory abducens ( $\mathrm{AP}=-15.5 \mathrm{~mm}, \mathrm{~L}=1.2 \mathrm{~mm}$; $\mathrm{D}=18 \mathrm{~mm}$ from cerebellum surface) nuclei. When necessary, the targeted stimulating sites were approached at AP angles. The final position of these stimulating electrodes was determined by the eyelid or eye retraction movements evoked by a pair of square $(50 \mu \mathrm{s})$ pulses $(1 \mathrm{~ms}$ interpulse interval and $0.5-2 \mathrm{~mA}$ ) applied to the corresponding electrode.

In addition, selected animals were implanted with stimulating electrodes or with guide cannulae in the ipsilateral $\mathrm{MC}(\mathrm{AP}=2 \mathrm{~mm}, \mathrm{~L}=2$ $\mathrm{mm} ; \mathrm{D}=1 \mathrm{~mm}$ from brain surface), the contralateral cerebellar IP nucleus ( $\mathrm{AP}=-19 \mathrm{~mm}, \mathrm{~L}=3 \mathrm{~mm} ; \mathrm{D}=6 \mathrm{~mm}$ from cerebellum surface), or the ipsilateral $\mathrm{RN}(\mathrm{AP}=8.5 \mathrm{~mm}, \mathrm{~L}=1.5 \mathrm{~mm}, \mathrm{D}=10 \mathrm{~mm})$. Guide cannulae were implanted to a depth of up to $1 \mathrm{~mm}$ above the selected site. Stimulating electrodes were made from $200 \mu \mathrm{m}$ enamelcoated silver wire. Guide cannulae consisted of a 21 gauge stainless steel needle protected by a removable 25 gauge stainless steel rod during nonrecording periods.

All the animals were prepared for the classical conditioning of eyelid responses (see Fig. $3 A, B$ ). For this, animals were implanted with recording bipolar hook electrodes in the left $\mathrm{OO}$ muscle. These electrodes were made from multistranded, Teflon-coated stainless-steel wire (A-M Systems) with a total external diameter of $230 \mu \mathrm{m}$ and bared $\sim 1 \mathrm{~mm}$ at the tip. A head-holding system, consisting of three bolts cemented to the skull perpendicular to the horizontal stereotaxic plane, was also implanted. All stimulating and recording electrodes were connected to a 9-pin socket attached to the holding system.

Recording and stimulating procedures. Recording sessions began 1 week after surgery. The experimental animal was placed in a Perspex restrainer designed for limiting its movements (Gruart et al., 2000). The restraining box was placed on the recording table and was surrounded by a black cloth. The recording room was kept softly illuminated, and a $50 \mathrm{~dB}$ background white noise was switched on during the experiments. For all the subjects, the first two recording sessions consisted of adapting the rabbit to the restrainer and to the experimental conditions; no stimulus was presented during these two sessions.

The EMG activity of the OO muscle was recorded with Grass P511 differential amplifiers with a bandwidth of $0.1 \mathrm{~Hz}$ to $10 \mathrm{kHz}$ (GrassTelefactor). Neuronal electrical activity was recorded in the RN area with a NEX-1 preamplifier (Biomedical Engineering). These recordings were performed with glass micropipettes filled with $2 \mathrm{M} \mathrm{NaCl} \mathrm{(3-5} \mathrm{M} \Omega$ resistance) and filtered in a bandwidth of $1 \mathrm{~Hz}$ to $10 \mathrm{kHz}$. Field potentials were recorded with low-resistance electrodes (1-3 $M \Omega$ ) or lowpass filtered $(>100 \mathrm{~Hz})$ from unitary recordings. The recording area was approached with the help of stereotaxic coordinates (Girgis and Shih-Chang, 1981), and of the antidromic and synaptic field potentials evoked by single or double ( $1 \mathrm{~ms}$ interpulse interval) pulses presented to the implanted stimulating electrodes (Fig. $1 B-G$ ). Electrical stimulation was achieved across an ISU-220 isolation unit attached to a programmable CS-220 stimulator (Cibertec). Criteria to determine whether the recorded and the activated neuron were the same, and to discriminate somatic versus axonic recordings, were systematically followed (Múnera et al., 2001). The recording micropipette was always removed at the end of each recording session and the recording chamber sterilized and closed.

Tones were applied from a loudspeaker located $80 \mathrm{~cm}$ below the animal's head. Air puffs were applied through the opening of a plastic pipette $(3 \mathrm{~mm}$ in diameter) attached to a metal holder fixed to the animal-holding system (dual-channel air-puff device; Biomedical Engineering).

Classical eyeblink conditioning. Conditioning consisted of two habituation and six conditioning sessions. A delay (tone/air puff) conditioning paradigm was used. The CS consisted of a $350 \mathrm{~ms}, 600 \mathrm{~Hz}, 85 \mathrm{~dB}$ tone. The US started $250 \mathrm{~ms}$ after CS onset, and consisted of a $100 \mathrm{~ms}, 3 \mathrm{~kg} / \mathrm{cm}^{2}$ air puff directed at the left cornea. The unconditioned stimulus (US) coterminated with the CS. Conditioning sessions consisted of 66 trials ( 6 series of 11 trials each) separated at random by intervals of 50-70 s. Of the 66 trials, 10\% were test trials in which the CS was presented alone. A complete conditioning session lasted for $\sim 1 \mathrm{~h}$. The CS was presented alone during habituation sessions for the same number of blocks/session and trials/block. As criteria, we considered a "CR" the presence, during the CS-US interval, of electromyographic (EMG) activity lasting $>10 \mathrm{~ms}$ and initiated $>50 \mathrm{~ms}$ after CS onset. In addition, the integrated EMG activity recorded during the CS-US interval had to be at least 1.2 times greater than the integrated EMG recorded immediately before CS presentation (Gruart et al., 2000; Leal-Campanario et al., 2007).

Neural activity was recorded in the $\mathrm{RN}$ area during habituation and conditioning sessions (see Figs. 3-6). Neuron isolation and identification procedures were performed during the time intervals in which the pair of CS-US stimuli was not presented (Múnera et al., 2001). Usually, a range of 2-7 RN neurons were antidromically identified and recorded per conditioning session.

Drug microinjection. A 5\% solution of lidocaine (Sigma-Aldrich) was injected by means of a calibrated injection tube (30G), coupled to a $10 \mu \mathrm{l}$ Hamilton syringe, which was advanced through the guide tube. Drug solutions were constantly injected from $5 \mathrm{~min}$ before until the end of the conditioning session at a rate of $0.1 \mu \mathrm{l} / \mathrm{min}$ with the help of a microinfusion pump (310; KD Scientific). Two additional animals were injected in the IP nucleus with $2 \mu \mathrm{l}$ of a $1 \mathrm{mg} / \mathrm{ml}$ solution of muscimol (Sigma-Aldrich), dissolved in artificial CSF, pH 7.4 (Jiménez-Díaz et al., 2004), and at a rate of $0.1 \mu \mathrm{l} / \mathrm{min}$.

Histology. At the end of the experiments, animals were deeply anesthetized with sodium pentobarbital (50 mg/kg, i.p.), and perfused transcardially with saline and $4 \%$ paraformaldehyde (PFA). The proper location of eyelid EMG electrodes was checked. To determine the final location of recording sites, stimulating electrodes, and injection cannulae, the brain was removed and cut into slices $(50 \mu \mathrm{m})$, and the relevant brain areas were processed for Nissl (toluidine blue) staining (see Fig. $2 B-G$ ).

In accordance with previous descriptions (Mendez and Hong, 1997), the retrograde fluorescent tracer Fluorogold ( $2 \%$ dissolved in saline; $\mathrm{H} 22845$, Invitrogen) was used to localize RN neurons projecting to the facial nucleus (FN; Fig. $2 I-K$ ). Injections were performed in the FN of 
three additional animals with the help of a Hamilton microsyringe $(2 \mu 1$ in $10 \mathrm{~min}$ ). The injection site was approached previously by antidromic field potentials evoked in the FN by electrical stimulation of the ipsilateral facial nerve. After 1 week of survival time, animals were intracardially perfused with $4 \%$ PFA in PB buffer, $0.12 \mathrm{M}$. Frozen $50 \mu \mathrm{m}$ sections were coverslipped with Citifluor (Citifluor). Injection sites in the FN and retrograde-labeled cells in the RN were photographed, using a fluorescent microscope with the help of a filter of $365 \mathrm{~nm}$ excitation (Observer.Z1; Carl Zeiss MicroImaging).

Data collection and analysis. The unitary activity recorded in the RN area, the unrectified EMG activity of the recorded muscles, and 1-V rectangular pulses corresponding to CS, US, and electrical stimuli presented during the different experimental situations, were acquired online through an 8-channel analog-to-digital converter (CED 1401-plus; CED), and transferred to a computer for quantitative off-line analysis. Data were sampled at 25,000 Hz (for unitary recordings) or $5000 \mathrm{~Hz}$ (for EMG recordings), with an amplitude resolution of 12 bits. Computer programs (Spike2 and SIGAVG; CED) were used to display unitary and EMG activities. These programs allowed the quantification, with the aid of cursors, of the onset latency and area $(\mathrm{mV} \times \mathrm{s})$ of the rectified EMG activity of the OO muscle and the latency, peristimulus time histograms (PSTHs), and instantaneous firing rate of unitary recordings. The programs also allowed the representation of PSTHs and/or the firing rate of the recorded neurons (Múnera et al., 2001; Leal-Campanario et al., 2007). When necessary, PSTHs were converted to firing rate following this equation (Rieke et al., 1997): firing rate (spikes/s) $=($ No. of spikes per bin/No. of repetitions $) \times(1000 /$ bin size, in $\mathrm{ms})$.

Statistical analyses were performed using the Sigma Plot 11.0 package (Sigma Plot) for a statistical significance level of $p=0.05$. Mean values are followed by their SEM. Collected data were analyzed using the oneway or two-way ANOVA test, with time or session as repeated measure, coupled with contrast and/or nonparametric analysis when appropriate. Repeated-measures ANOVA allowed checking the statistical differences of the same group across sessions. The Student-Fisher $t$ test was used when necessary.

\section{Results}

\section{Identification of recorded $\mathrm{RN}$ and pararubral neurons}

As illustrated in Figure $1 A$, the $\mathrm{RN}$ was approached using a transcortical approach and following available stereotaxic coordinates (Girgis and Shih-Chang, 1981). Recorded neurons were identified by their antidromic activation from their projection sites (Robinson et al., 2001; Miller and Gibson, 2009): the contralateral facial (Fig. $1 B$ ) or the accessory abducens nuclei (Fig. $1 C$ ), and with the additional help of the collision test. Mean activation latencies were $0.92 \pm 0.03 \mathrm{~ms}$ (mean $\pm \mathrm{SEM} ; n=$ $109)$ from the FN and $1.01 \pm 0.04(n=35)$ from the accessory abducens nucleus, with no significant differences $(p=0.09$, Student's $t$ test). Additional support for the antidromic nature of spike activation is that it followed stimulation frequencies of up to $500 \mathrm{~Hz}$.

Identified RN neurons were easily activated (50 $\mu$ s square, 1 ms of interval paired pulses, $0.5-2 \mathrm{~mA}$ ) from the ipsilateral $\mathrm{MC}$ at $5 \pm 0.32 \mathrm{~ms}$ of latency (Fig. $1 D$ ) and from the contralateral cerebellar IP nucleus at a shorter time (1.82 $\pm 0.06 \mathrm{~ms}$; Fig. $1 E)$. Interestingly, the electrical stimulation of the $\mathrm{MC}$ also evoked late, variable activations of $\mathrm{RN}$ neurons, indicating the presence of local reverberating circuits (Horn et al., 2002). The implanted MC sites were clearly related with eyelid movements, because their stimulation evoked the short-latency $(9.4 \pm 0.2 \mathrm{~ms})$ activation of the EMG activity of the contralateral OO muscle, while the stimulation of the RN (50 $\mu$ s single, square pulses, $0.2-2 \mathrm{~mA}$ ) evoked responses of slightly shorter latencies $(7.5 \pm 0.2 \mathrm{~ms})$, indicating its disynaptic nature (Fig. $1 F$ ). Muscle activation from the IP nucleus (by a pair of pulses, $1 \mathrm{~ms}$ interval) took $10.1 \pm 0.3$ ms (data not shown). For comparison, OO muscle activation from the FN took place in $2.5 \mathrm{~ms}$ (Fig. $1 F$ ). The latency of spiketriggered averaged activities of FN and $\mathrm{OO}$ muscle further confirmed that RN neurons recorded and analyzed here project to the $\mathrm{FN}$ and have a disynaptic activation effect $(7.5 \pm 0.2 \mathrm{~ms})$ on this muscle (Fig. 1G).

Representative photomicrographs with the location of stimulating electrodes are illustrated in Figure $2 A-E$. Small electrolytic lesion marks made with tungsten electrodes at the end of the recording sessions indicated that $\mathrm{RN}$ neurons related to eyelid reflex and conditioned responses occupied a dorsolateral position in the rostral part of the nucleus (Fig. $2 F, G$ ). With the help of these electrolytic marks and collected information regarding stereotaxic coordinates, we made a precise reconstruction of the location of recorded and identified RN neurons $(n=144)$. Figure $2 \mathrm{H}$ indicates that recorded neurons (types $\mathrm{A}$ and $\mathrm{B}$; see below) also formed a cell column over the dorsolateral part of the RN (Desmond and Moore, 1991). To further confirm that these pararubral neurons were also involved in eyelid motor responses, we injected the retrograde tracer Fluorogold in the FN of two animals. As already reported in rats (Ruigrok and Cella, 1995; Morcuende et al., 2002) and cats (Pong et al., 2008), retrogradelabeled neurons were concentrated in the lateral aspect of the contralateral RN and in the dorsally adjoining pararubral area (Fig. 2I-K). Probably, those labeled neurons occupying more ventral positions in the $\mathrm{RN}$ correspond to type $\mathrm{C}$ and $\mathrm{E}$ neurons (see below).

\section{Neuronal response types}

Apart from some preliminary recording sessions in the absence of conditioning stimuli, performed to adapt the recording system to the experimental needs, red and pararubral nucleus neurons were recorded during the two habituation and the six conditioning sessions presented here to all of the conditioned animals. In Figure 3 the experimental procedures for unitary recording and for classical eyeblink conditioning are illustrated. For analysis, the raw EMG recordings (Fig. $3 B$ ) were rectified and averaged (Fig. $3 C, D$ ), while unitary firing data (Fig. $3 B$ ) were passed across a discrimination window and accumulated (bin size $=10 \mathrm{~ms}$ ) for (usually) 10 successive trials (Fig. 3D).

Recorded neurons were classified in five different groups (A-E) depending on their antidromic activation from the facial or the accessory abducens nucleus, their spontaneous firing, and their firing during the CS-US interval (Fig. 4).

Type A neurons (Figs. 3, 4A) were activated antidromically from the $\mathrm{FN}$ and presented an irregular spontaneous firing rate, with mean values ranging from 10 to 30 spikes/s $(n=55)$. Type A neurons fired a burst of action potentials preceding $(\approx 10-40$ $\mathrm{ms}$ ) the EMG activation of the OO muscle during the CS-US interval, i.e., during the generation of the CR. In the presence of CRs, type A neurons showed peak firing reaching 500 spikes/s (Fig. $4 A$, right histogram). Characteristically, type A neurons decreased their firing rate immediately after the paired CS-US presentation (Fig. $3 B, C$ ). Type B neurons (Fig. $4 B$ ) were activated antidromically from the facial $(n=29)$ or the accessory abducens nucleus $(n=17)$. They showed a higher $(40-60$ spikes/s) and more stable spontaneous firing rate than type A cells, but were equally activated in the presence of CRs, reaching high peak firing rates during the CS-US interval (Fig. $4 B$, right histogram). In addition, type $\mathrm{A}$ and $\mathrm{B}$ neurons were easily activated by different types of stimulus, such as air puffs directed at the face, tones, and flashes of light, indicating that they received not only motor commands, but also information of different sensory modalities (Padel et al., 1988). Because of their firing properties, type A and B neurons 


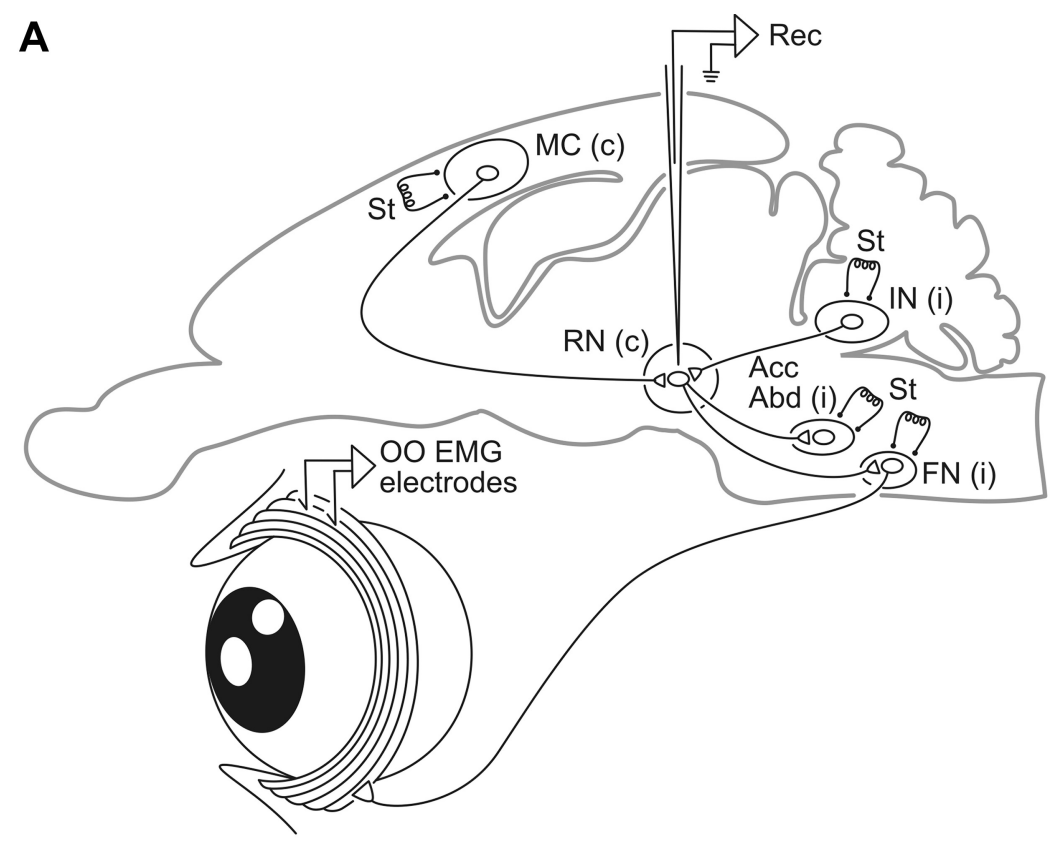

B

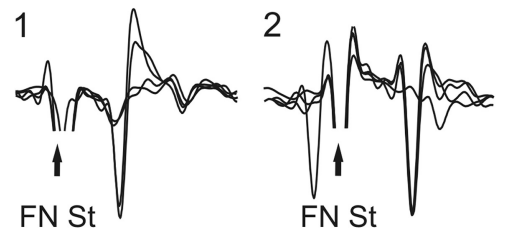

D

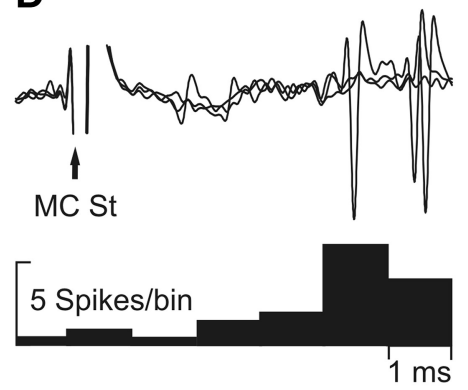

$\mathbf{F}$
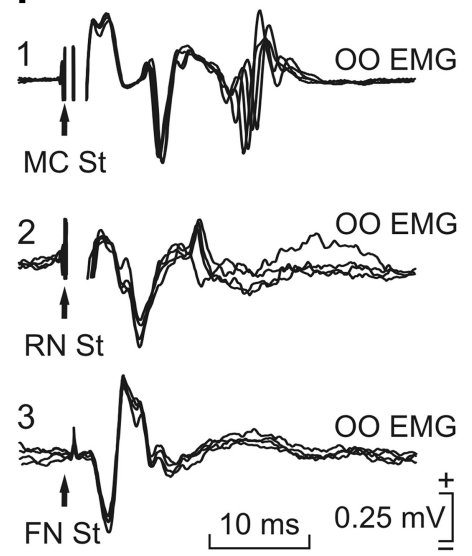

1

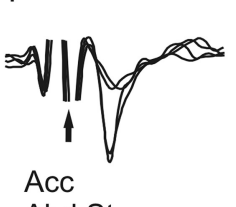

Abd St

E

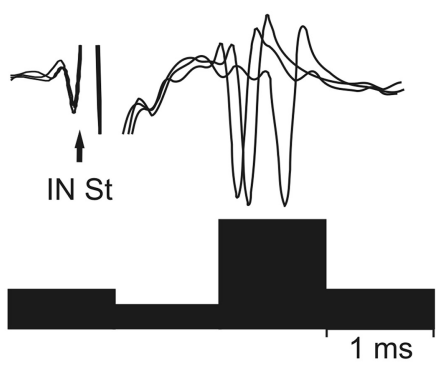

\section{G}

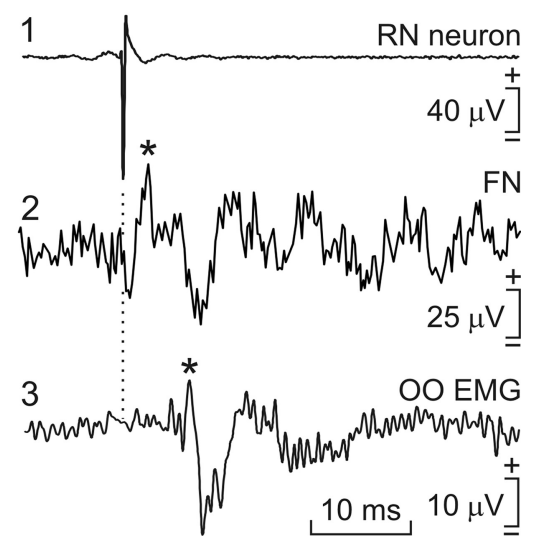

were considered to be directly involved in the acquisition of classically conditioned eyelid responses, and were processed for further quantitative analysis.

Type $\mathrm{C}$ neurons were activated antidromically only from the FN $(n=25)$. Like type B neurons, they presented a high-frequency $(30-60$ spikes/s) tonic firing rate, but, in contrast, they decreased $(-25.6 \pm 3.8 \%$ on average $)$ their firing during the CS-US interval in simultaneity with the presence of CRs (Fig. 4C). For the following reasons, we assumed that these $\mathrm{RN}$ neurons were probably projecting to masticator muscles: (1) they were exclusively activated from the $\mathrm{FN}$; (2) the firing rate of type $\mathrm{C}$ neurons did not change (i.e., increase or decrease) across the successive conditioning sessions; and (3) they were inhibited when type A and B neurons increased their firing rate, suggesting that type $\mathrm{C}$ neurons were involved in the already described antagonist activities between the zygomatic and buccal branches of the facial nerve (Pauletti et al., 1993; Godefroy et al., 1998; Gruart et al., 2003). Type D neurons $(n=18)$ were activated antidromically only from the accessory abducens nucleus. They presented a high spontaneous firing rate that was increased during CS-US presentations, but without a definite temporal relationship (Fig. 4D). In fact, their discharge rates look like a particular case of type B neurons. Finally, type E neurons $(n=25)$ were recorded in the same red and pararubral areas as the other four types, but they could not be activated antidromically and did not modify their firing rates during reflex or conditioned eyeblinks. Most of these neurons responded to neck and/or body movements of the recorded animal, suggesting that they were in fact rubrospinal neurons involved in the activation of skeletal muscles (Eccles et

Figure 1. Experimental design and identification of recorded RN neurons. $\boldsymbol{A}$, Diagrammatic representation of the experimental design. RN neurons contralateral [RN (c)] to the left eye were recorded using a transcranial cortex approach (Rec). Stimulating (St) electrodes were implanted in the ipsilateral facial [FN (i)], accessory abducens [Acc Abd (i)], and cerebellar interpositus [IN (i)]

$\leftarrow$

nuclei, and in the contralateral eyelid area of the $M C$ (c). The EMG activity of the 00 muscle was also recorded. $\boldsymbol{B}, \boldsymbol{C}$, Antidromic activation of RN neurons from the FN $(B, 1)$ or the Acc Abd nucleus $(\boldsymbol{C}, 1)$ at threshold straddling intensities. The arrows indicate the stimulus artifacts. Representative examples of the collision test for RN neurons activated from the $F N(B, 2)$ and the Acc Abd nucleus (C, 2) are also shown. $\boldsymbol{D}, \boldsymbol{E}$, Synaptic activation of RN neurons from the MC $(\boldsymbol{D})$ and the IN nucleus (E). The bottom histograms illustrate PSTHs ( $n=5$ times; bin size $=1 \mathrm{~ms}$ ). $\boldsymbol{F}$, From top to bottom are illustrated the EMG activity evoked in the 00 muscle by double (1 ms interval) pulses applied to the MC (1) and to the RN (2), and of single pulses presented to the FN (3). G, Spike-triggered extracellular activity (asterisks) recorded in the FN (2) and in the 00 muscle (3). The triggering action potential corresponded to an antidromically identified RN neuron (1). Average was repeated 1000 times. Calibrations in $\boldsymbol{C}$ are also for $\boldsymbol{B}$, and the one in $\boldsymbol{D}$ is also for $\boldsymbol{E}$. 


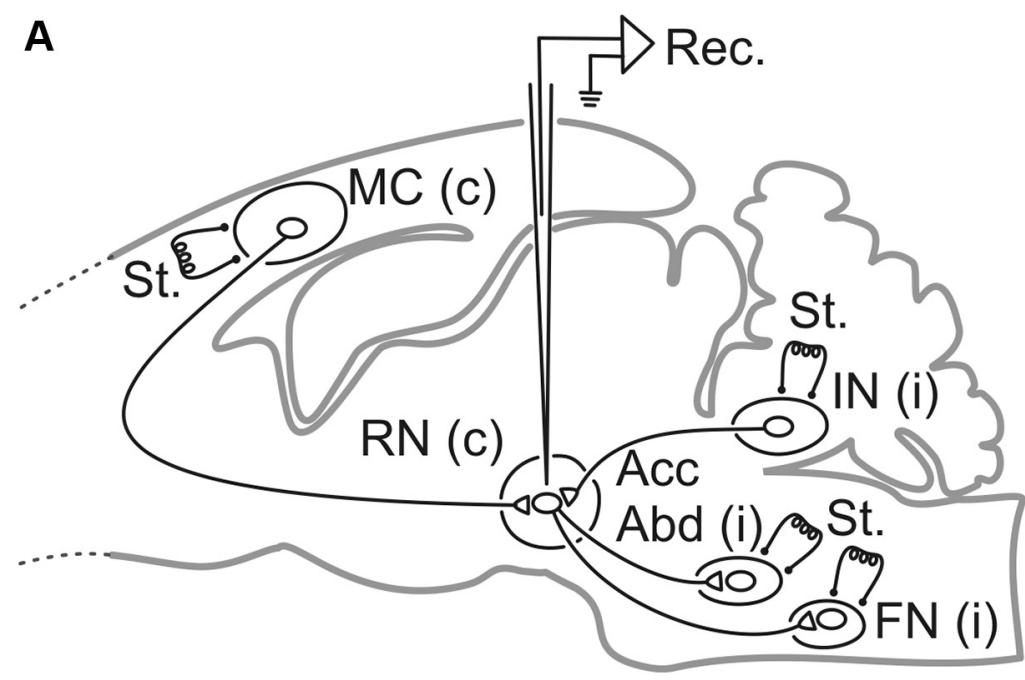

B
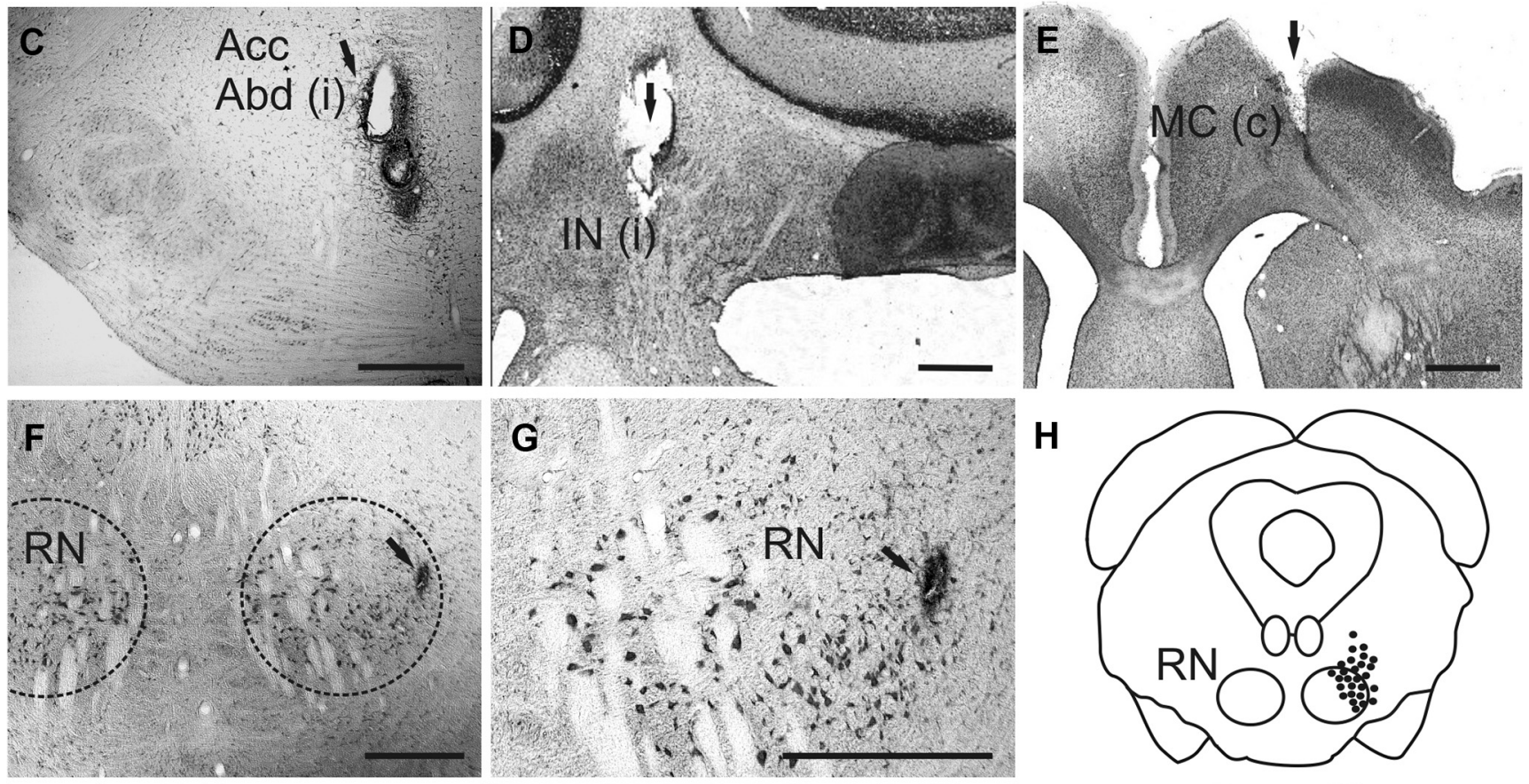

$\mathbf{H}$
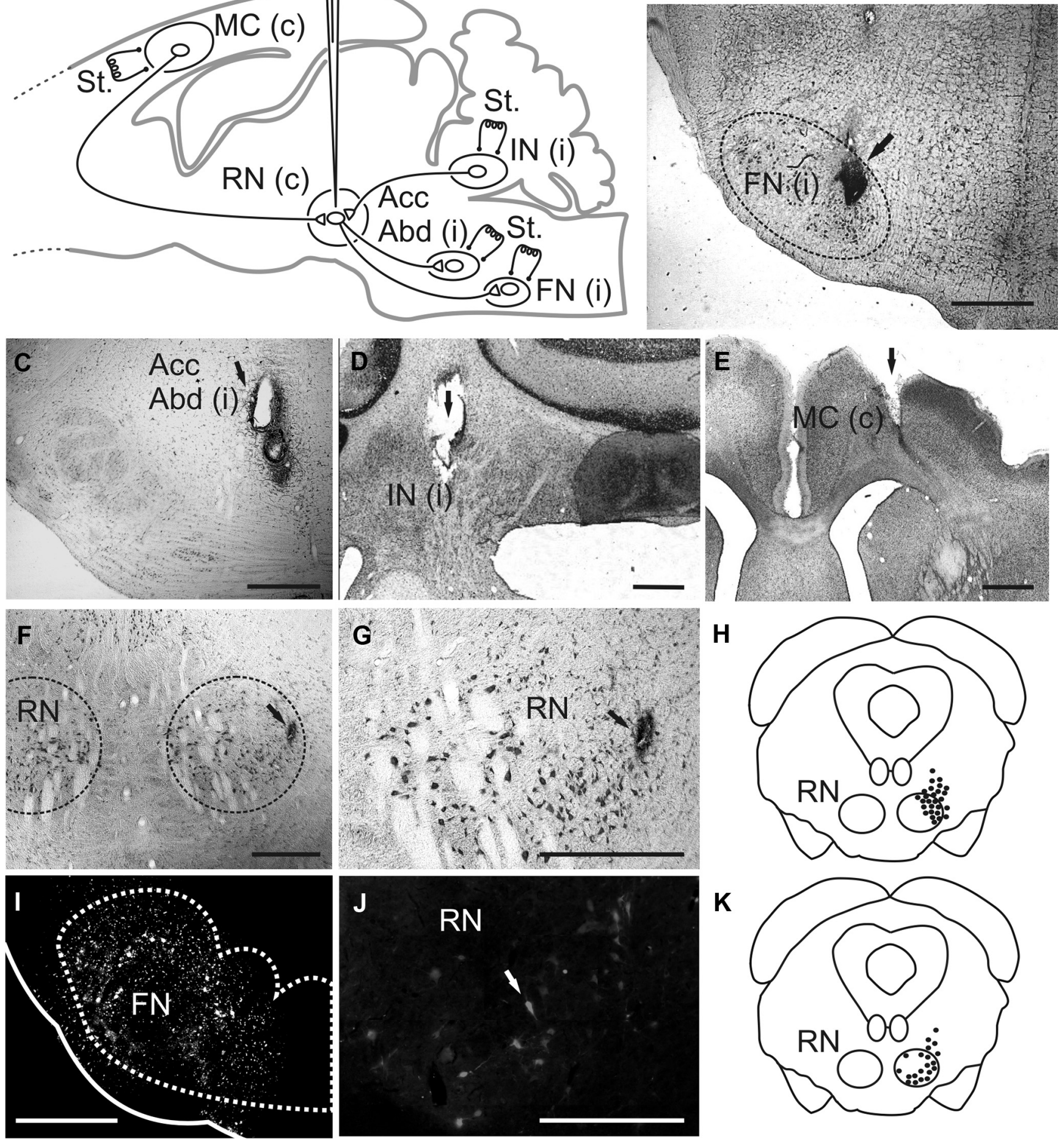

Figure 2. Location of recording and stimulating electrodes. $A$, Diagram illustrating the differentstimulating and recording sites. Abbreviations are as defined in the legend to Figure $1 . B-E$, , Photomicrographs of different brain coronal sections illustrating electrolytic marks (arrows) made with stimulating electrodes implanted in the FN ( $\boldsymbol{B})$, the $\operatorname{Acc} A \operatorname{Abd}(\boldsymbol{C})$ and the IN (D) nuclei, and the MC (E). $\boldsymbol{F}, \boldsymbol{G}$, Photomicrographs of coronal sections through the midbrain illustrating Nissl-stained RN neurons and a small electrolytic mark corresponding to the recording area. $I$, A photomicrograph of Fluorogold injection site in the FN.J, A photomicrograph illustrating labeled neurons located in the RN following Fluorogold injection in the contralateral FN. $\boldsymbol{H}$, Reconstruction of the location of recording RN neurons. Each black circle represents five recorded neurons. $\boldsymbol{K}$, Map reconstruction of RN and pararubral neurons (black circles) projecting to the FN as indicated by their Fluorogold labeling. Each black circle represents five labeled neurons. Abbreviations: c, i, contra- and ipsilateral. Calibration bars: $1 \mathrm{~mm}$. 
al., 1975; Huisman et al., 1981, 1982). Because of their lack of specificity in relation to the evolution and profiles of CRs, RN neurons classified as $\mathrm{C}-\mathrm{E}$ were not included in the subsequent analysis.

\section{Changes in the activity of $\mathrm{RN}$ and pararubral neurons during the acquisition of conditioned eyeblink responses}

The top graph of Figure $5 A$ illustrates the learning curve of classically conditioned rabbits. As already reported for the same species using similar training paradigms (Gruart et al., 2000; Leal-Campanario et al., 2007), conditioned animals started to increase their percentage of conditioned responses significantly $\left(F_{(7,14)}=6.83 ; p<\right.$ $0.001)$ by the third conditioning session $(48.2 \pm 15.5 \%)$, reaching asymptotic values $(86.7 \pm 5.5 \%)$ by the fifth conditioning session. The rectified area $(\mathrm{mV} \times \mathrm{s})$ of the EMG activity generated by the OO muscle during the CS-US interval (Fig. $5 A$, middle graph) also increased across training, reaching significantly $(p<0.001$; $\chi^{2}=38.56$ ) larger values from the second to the sixth conditioning sessions. The increase was $36.7 \pm 4.2 \%$ with respect to baseline (habituation) values by the second conditioning session. Interestingly, peak values were reached in the fourth and fifth sessions $(\approx 80 \%)$, but those values decreased for the sixth session $(59.9 \pm 6.7 \%)$. Changes in neuronal firing across training paralleled those observed for the integrated EMG activity of the OO muscle (Fig. $5 \mathrm{~A}$, bottom graph): the mean firing activity of recorded type $\mathrm{A}$ and $\mathrm{B}$ neurons $(n=75 ; n \leq$ 10 per session $)$ showed a significant $\left(p<0.001 ; \chi^{2}=422.37\right)$ increase in rate with respect to baseline values by the second session and reached peak values by the fourth. As in the case of the EMG area, the mean firing activity of identified $\mathrm{RN}$ and pararubral neurons started to decrease by the fifth conditioning session.

An attempt was made to determine the linear relationships between those three variables included in Figure $5 A$; namely, the percentage of CRs, the EMG area during the CS-US interval, and the mean firing rate during the same period of time (Fig. 2 B). As illustrated in Figure $5 C$, although the three variables were linearly related, the best linear fit $(r=0.92 ; p<0.01)$ found was for the relationships between the mean firing rate of type A and $B$ neurons and the rectified EMG area, while the lowest value was for the relationship between the percentage of CRs and the mean firing rate $(r=0.7 ; p<0.05)$. Finally, the linear relationships between the percentage of CRs and the rectified EMG area had intermediate values $(r=0.81 ; p<0.05)$. Together, these results suggest that the firing of RN neurons is related more to changes in eyelid motor performance during the training than to the precise evolution of the acquisition process (i.e., the learning curve).

Effects of acute inactivation of the MC on the acquisition of classical eyeblink conditioning and on the firing properties of related $\mathrm{RN}$ neurons

It is known that the $\mathrm{RN}$ receives a significant collateral projection from descending pathways originated in the ipsilateral facial MC (Tsukahara et al., 1967; Pong et al., 2008; Miller and Gibson,
2009). As shown here, the electrical stimulation of the facial area of the MC evokes a short-latency, potent activation of RN neurons related to eyelid responses (Fig. 1D). In a first series of experiments, we studied the effects on MC inactivation (by a local injection of lidocaine) on the acquisition of eyelid CRs (Fig. 6A) in controls and injected animals ( $n=3$ per group). As shown in Figure $6 B$, lidocaine infusion into the MC during the fourth and fifth conditioning sessions reduced the percentage of CRs by $\sim 50 \%\left(F_{(7,14)}=1.606 ; p=0.006\right)$.

In a subsequent experiment, we decided to study the effects of MC inactivation across all conditioning sessions at the same time we recorded the evoked changes in the discharge rate of identified $\mathrm{RN}$ and pararubral type $\mathrm{A}$ and $\mathrm{B}$ neurons. In Figure $6 C$ the rectified and averaged $(n=66)$ EMG activity of the OO muscle corresponding to representative vehicle-injected (left set of records) and lidocaine-injected (right set of records) rabbits across the sixth conditioning sessions are illustrated. It should be noticed that lidocaine injection greatly reduced the expression of CRs, with a minor effect on the performance of the unconditioned responses. In fact, CRs in lidocaine-injected animals only started to appear by the fourth conditioning session, well after their appearance in the control animal.

A quantitative analysis of the evolution of CRs, of the rectified EMG area, and of the mean firing rate during the CS-US interval, is illustrated in Figure 6D. As shown, lidocaine infusion into the MC during the six conditioning sessions significantly reduced the percentage of CRs $\left(F_{(7,14)}=16.05 ; p<0.05\right)$, as well as the area of the EMG activity of the OO muscle $\left(F_{(7,35)}=4.12 ; p<0.05\right)$. In fact, in lidocaine-injected rabbits $(n=3)$ the two variables only started to reach a $>20 \%$ increase over habituation values by the fourth conditioning session. The mean firing rate of $\mathrm{RN}$ neurons evoked in lidocaine-injected animals was significantly lower than 

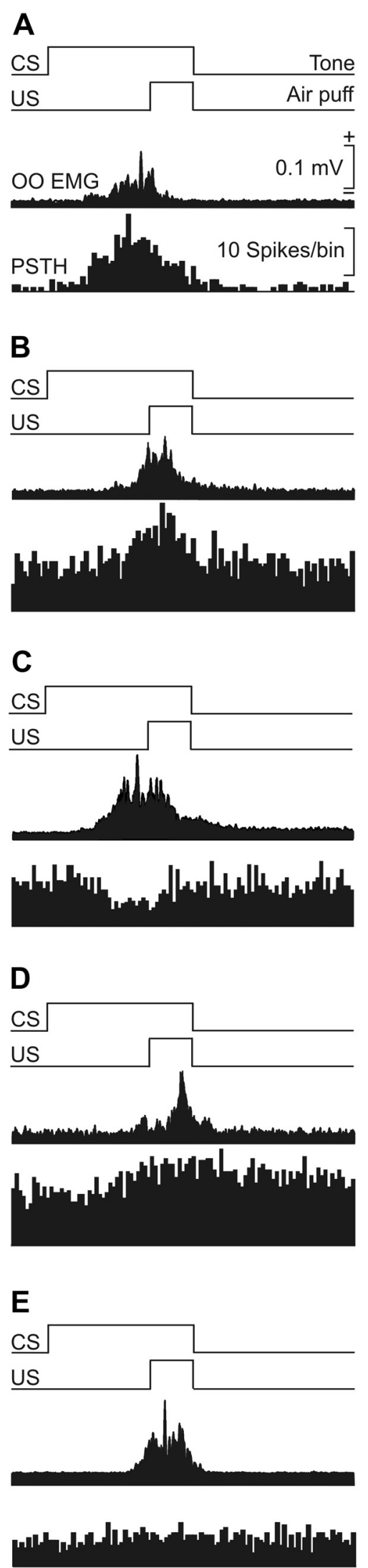

$0.2 \mathrm{~s}$
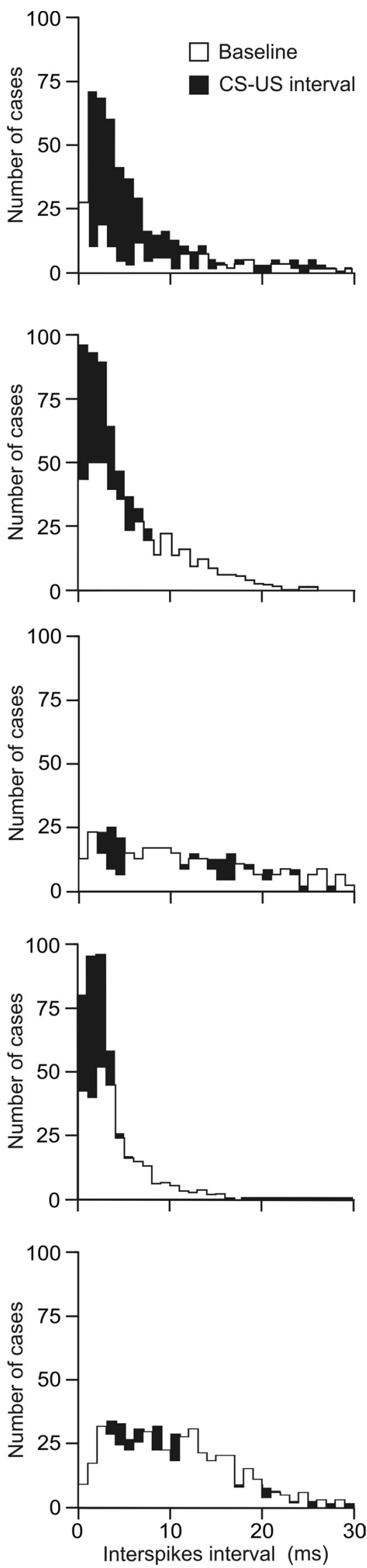

Figure 4. Representative examples of the different types of identified RN neuron recorded during classical conditioning of eyelid responses. All recordings were performed during the fourth conditioning session and were averaged from 10 successive trials. $A$, Left, Rectified and averaged EMG activity of the 00 muscle and the PSTH ( $n=10$ times; bin size $=10 \mathrm{~ms}$ ) of the firing control values $\left(p<0.001 ; \chi^{2}=161.653\right)$ Interestingly, the firing rate of $\mathrm{RN}$ neurons recorded in lidocaine-injected rabbits presented a U shape (Fig. $6 \mathrm{D}$, bottom graph), because it decreased from the first to the third conditioning sessions (as opposed to the increase in firing rate taking place in control rabbits), but it increased from the fourth to the sixth conditioning sessions, in parallel with the increase observed in the rectified EMG area.

On the whole, these results indicate that the MC plays an important role in the proper and timed generation of CRs in behaving rabbits, with a minor effect on unconditioned eyelid responses. In addition, the delayed initiation of CRs in lidocaine-injected animals could be the result of learning-dependent changes in neuronal firing activities taking place in the ipsilateral RN.

It is generally accepted that delay conditioning can be acquired by decerebrated and decorticated animals (Oakley and Russell, 1972; Ivkovich and Thompson, 1997; Case et al., 2002; Kotani et al., 2002). Indeed, those chronic lesions will allow the activation of hierarchically lower centers, as the RN and the pararubral areas. In opposition to this experimental approach, we performed here an acute inactivation of the MC with functional effects restricted to the conditioning session. In this regard, there are early reports indicating a reversible effect on the expression of CRs by the transient inactivation of cortical circuits (Megirian and Bures, 1970). Thus, transient and short-term inactivation of cortical motor centers will prevent the activation of other brain centers with learning capabilities. But, and as explained below (see Fig. $8 \mathrm{~B}$ ), the $\mathrm{RN}$ and the pararubral areas showed learningrelated changes in firing activities if cortical inactivation is performed in successive conditioning sessions.

Effects of acute inactivation of the cerebellar IP nucleus on the acquisition of classical eyeblink conditioning and on the firing properties of related $\mathrm{RN}$ neurons

As reported years ago (Tsukahara et al., 1967; Eccles et al., 1975; Canedo and Lamas, 1989) and further confirmed in Figure $1 E$, the electrical stimulation of the

$\longleftarrow$

activity of a type A neuron. Right, The interspike-interval distribution of the same neuron during spontaneous activity (baseline) and during the CS-US interval. $\boldsymbol{B}-\boldsymbol{E}$, Same as in $\boldsymbol{A}$, for neurons type $\boldsymbol{B}-\boldsymbol{E}$. 


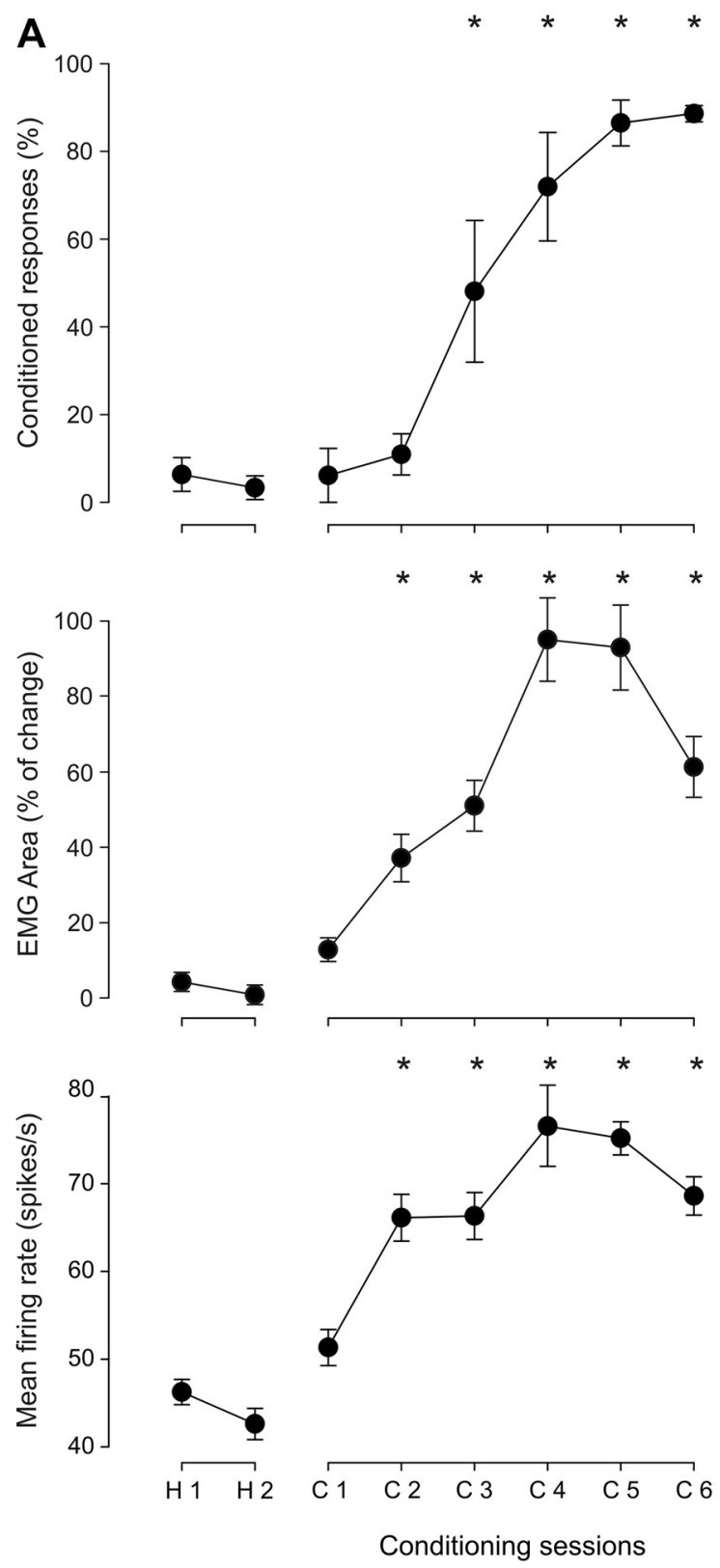

B
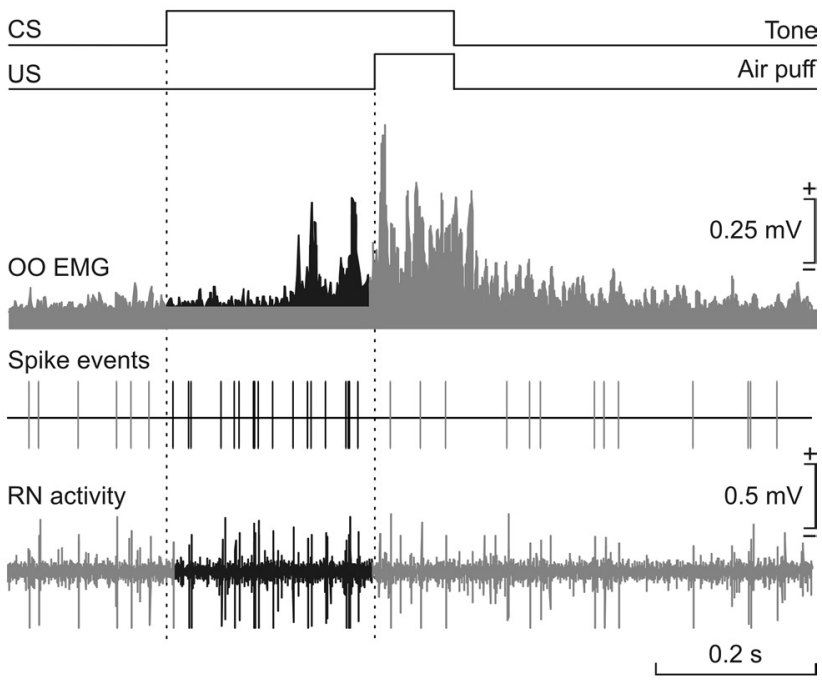

C

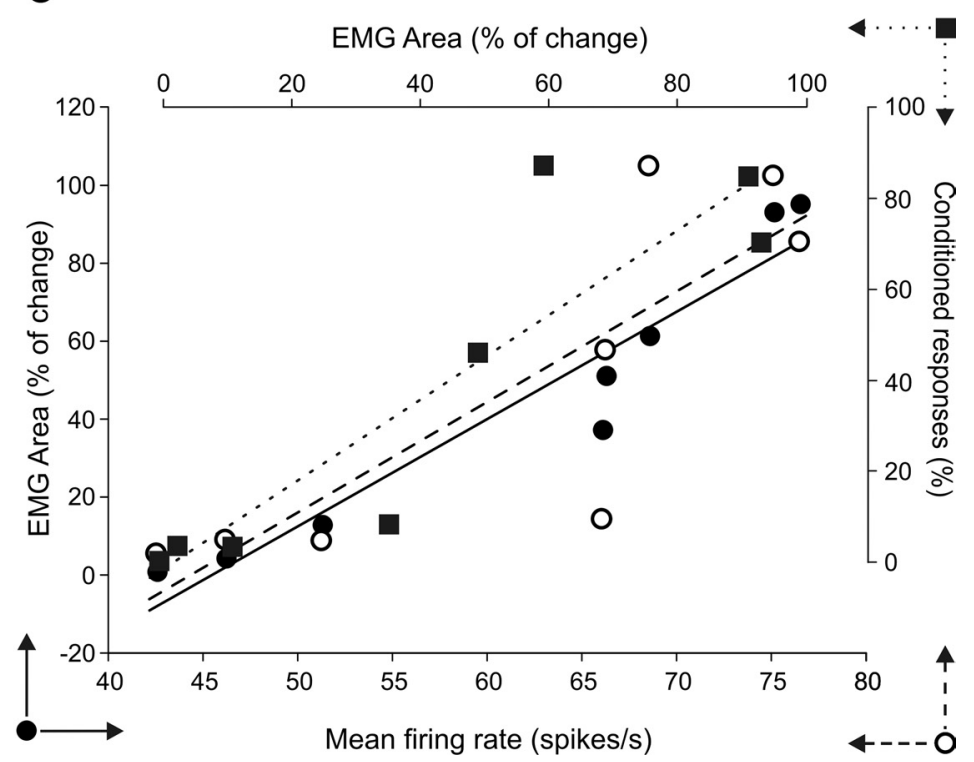

Figure 5. Learning curves and changes in the firing rate of RN neurons. A, The three graphs show the evolution of the percentage (\%) of CRs (top graph), of the $00 \mathrm{EMG}$ area ( $\mathrm{mV} \times \mathrm{s}$, in \% of change, middle graph), and of the mean firing rate (in spikes/s) of identified RN neurons recorded during the indicated sessions (bottom graph). Data were collected from four animals and for 75 identified neurons ( $n \leq 10$ per session). ${ }^{*} p<0.001$, one-way ANOVA. B, Selection of data corresponding to the increase in EMG area and in neuronal firing taking place during the CS-US interval. The selected changes in activity are indicated in black. C, Linear relationships between mean firing rate of type $A$ and $B$ RN neurons and the EMG area of the 00 muscle (black circles and continuous line; $r=0.92 ; p<0.01$ ), EMG area and the percentage of (Rs (black squares and dotted line; $r=0.81 ; p<0.01$ ), and mean firing rate and (Rs (white circles and dashed line; $r=0.7 ; p<0.05$ ).

contralateral cerebellar IP nucleus evokes a monosynaptic activation of RN neurons related to eyelid responses. In consequence, we also studied the effects of lidocaine infusion into the IP nucleus during classical conditioning of eyelid responses and simultaneously with recording the firing activities of RN neurons related to eyelid movements (Fig. 7A). Interestingly, lidocaine infusion into the IP nucleus evoked a rapid $(<200 \mathrm{~s})$ increase in the spontaneous firing rate of RN neurons (Fig. $7 B$ ), an effect not observed during lidocaine infusion into the MC. Since this enhanced neuronal firing could be ascribed to an unwanted effect of lidocaine on terminal axons of Purkinje cells, two additional animals were infused with muscimol (a potent agonist of $\mathrm{GABA}_{\mathrm{A}}$ receptors). In the two cases, the local microinjection of muscimol evoked a similar unspecific increase in $\mathrm{RN}$ firing rates.
In Figure $7 C$ the rectified and averaged $(n=66)$ EMG activity of the $\mathrm{OO}$ muscle corresponding to representative vehicle-injected (left) and lidocaine-injected (right) rabbits across the six conditioning sessions are illustrated. In contrast to results collected during lidocaine injections in the MC (Fig. $6 C)$, lidocaine infusion into the IP nucleus almost completely canceled out the expression of both conditioned and unconditioned eyelid responses (Fig. 7C). In fact, CRs in lidocaineinjected animals did not reach significant values for the six conditioning sessions. As already reported in behaving cats (Jiménez-Díaz et al., 2004) muscimol injections in the IP nucleus also significantly decreased $(p<0.001)$ the expression of both conditioned $(\geq 35 \%)$ and unconditioned $(\geq 35 \%)$ eyelid responses. 


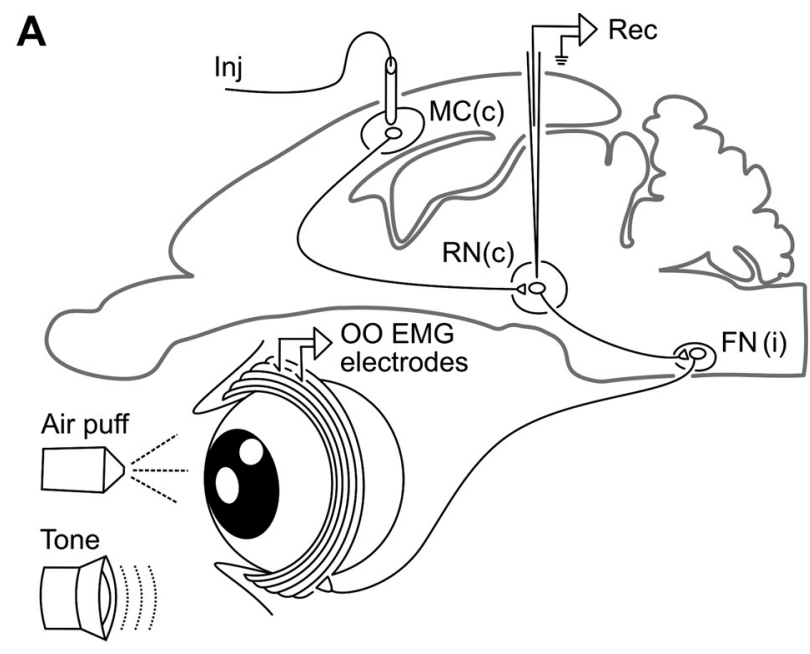

B

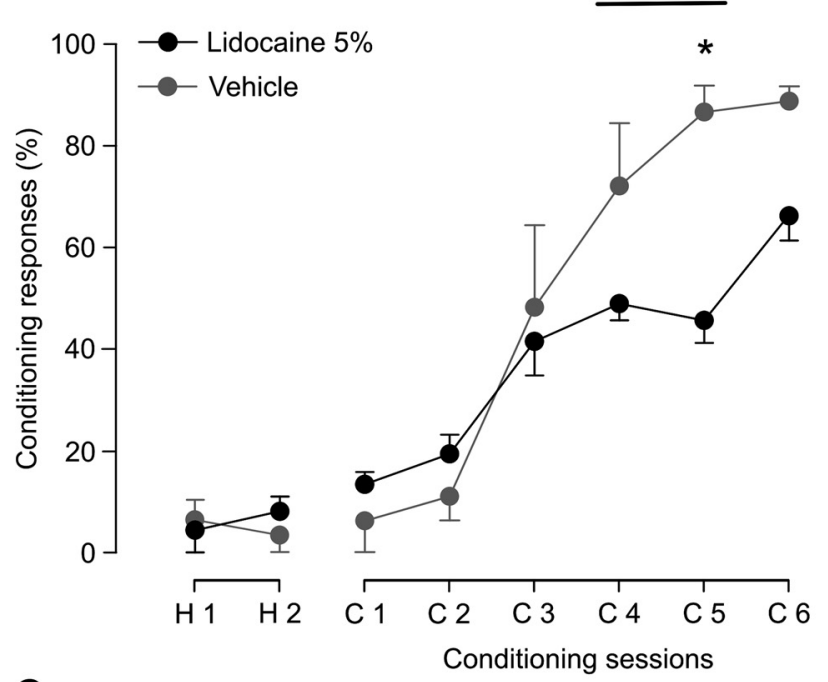

C

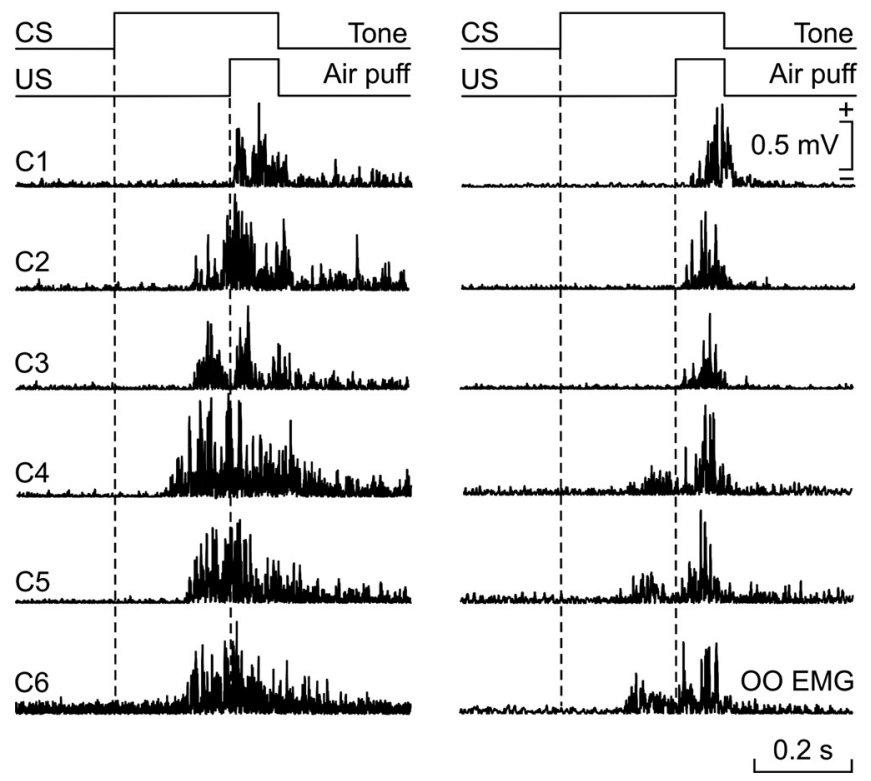

D
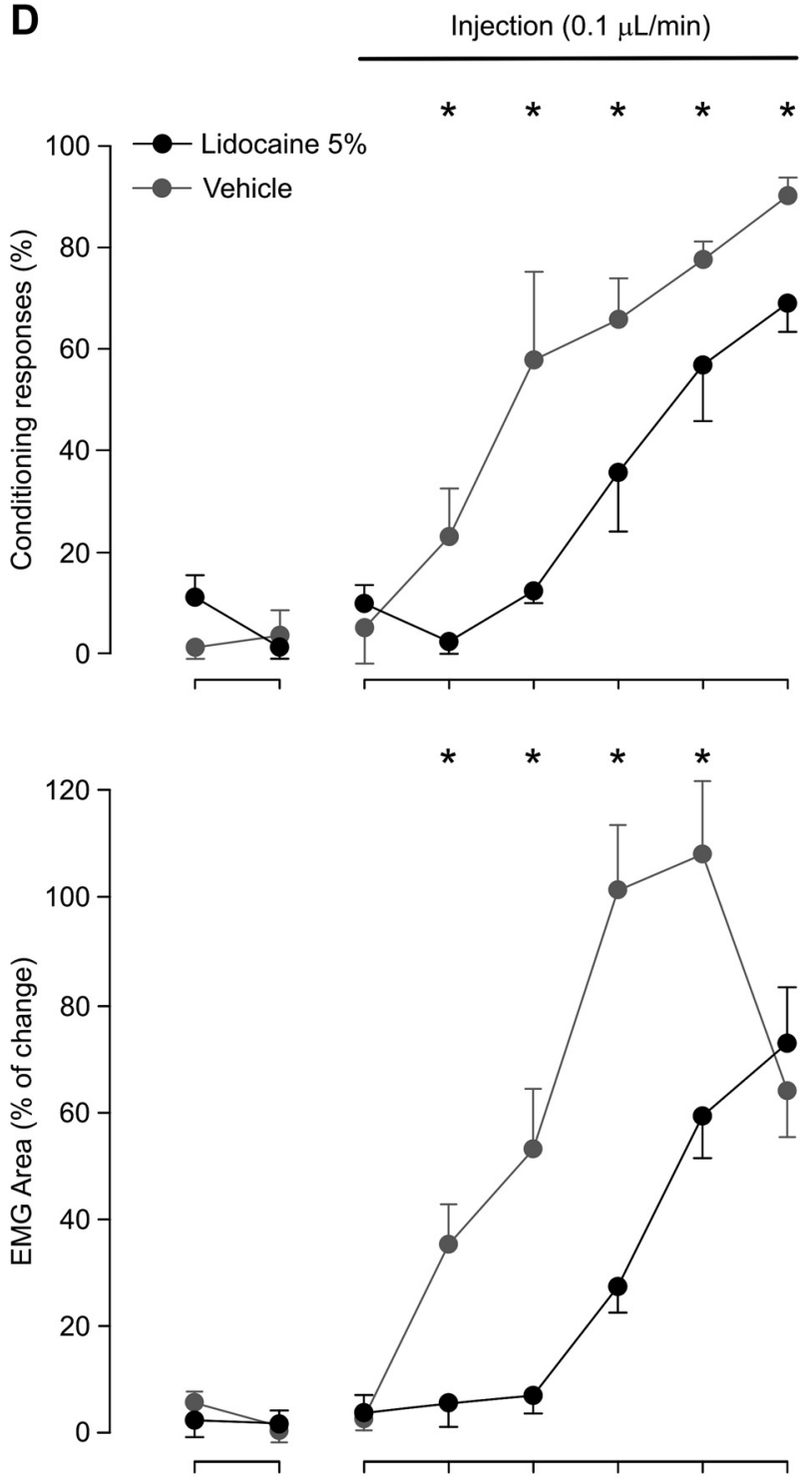

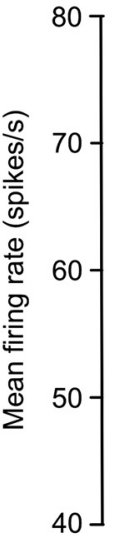
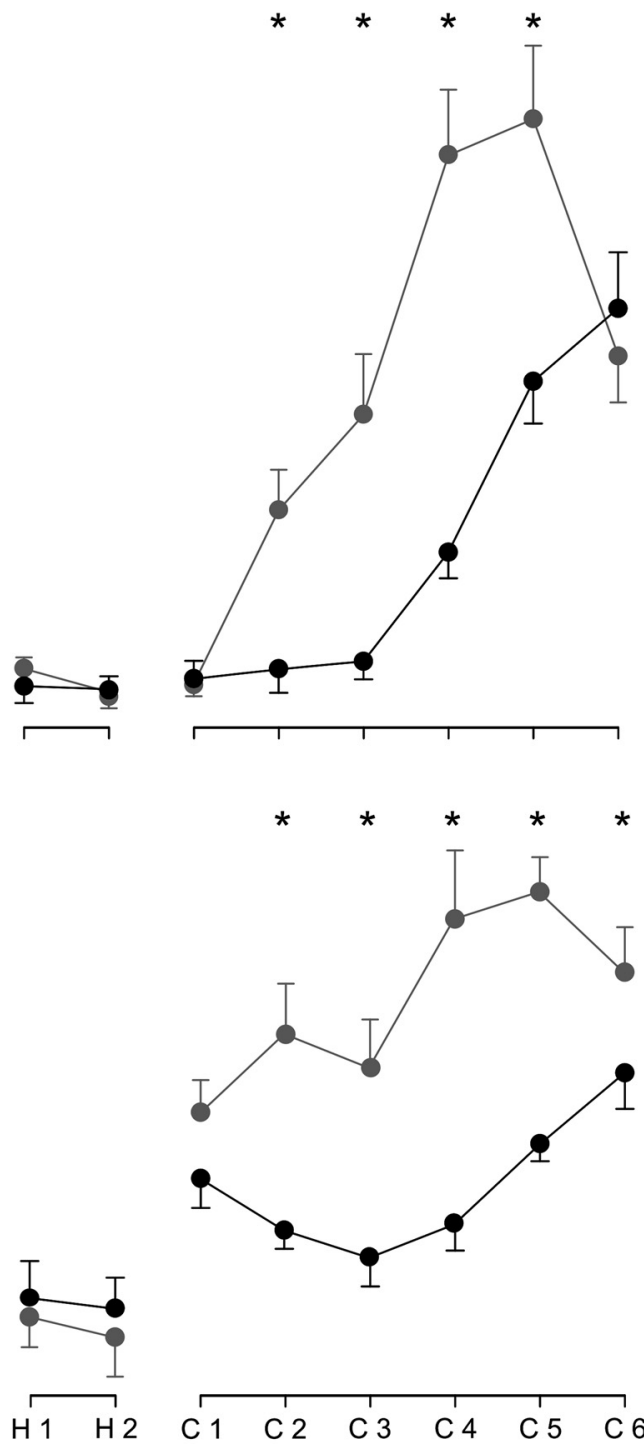

$\mathrm{H} 1 \mathrm{H} 2$

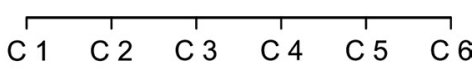

Conditioning sessions

Figure 6. Effects of $M C$ inactivation on learning curves and on the firing rate of RN neurons. $A$, Experimental design. The ipsilateral $M C$ was perfused with lidocaine ( $5 \%$ solution at a rate of 0.1 $\mu \mathrm{l} / \mathrm{min}$ ) or vehicle (Inj) during classical eyeblink conditioning and unitary recordings (Rec) of RN neurons. B, Quantitative analysis of data collected from three vehicle-injected (gray circles and lines) and three lidocaine-injected (black circles and lines) animals (mean \pm SEM). In this case, lidocaine was injected only during sessions 4 and 5 . Note the significant decrease in the percentage of (Rs $(p<0.005$; two-way ANOVA), C, Evolution of the rectified EMG activity of the 00 muscle across conditioning sessions in representative animals injected with vehicle (Figure legend continues.) 
A quantitative analysis of the evolution of CRs, of the rectified EMG area, and of the mean firing rate during the CS-US interval was also performed in rabbits injected with lidocaine in the IP nucleus (Fig. 7D). In this case, lidocaine infusion into the IP nucleus during the six conditioning sessions almost completely prevented the appearance of $\operatorname{CRs}\left(F_{(7,14)}=182.22 ; p<0.005\right)$, as well as reducing the area of the EMG activity of the OO muscle $\left(F_{(7,35)}=13.32 ; p<0.01\right)$. In fact, in lidocaine-injected rabbits $(n=3)$ the two variables never reached a $>20 \%$ increase over habituation values across the whole conditioning. Although no significant changes were observed in the mean firing rate presented by the two groups, the mean firing rate of RN neurons evoked in lidocaine-injected animals showed a significant $(p<$ 0.05 ) increase during the first conditioning session (Fig. $7 D$, bottom graphs). The firing rate of RN neurons of lidocaine-injected animals decreased progressively until reaching a minimum during the fourth training session. For the fifth and sixth conditioning sessions, the RN neurons of lidocaine-injected animals presented values similar to those of control rabbits.

We also checked the rate of recovery of CRs during the two conditioning sessions following lidocaine inactivation of the IP nucleus (Fig. 8C). The aim was to determine whether the inactivation of the IP nucleus prevented the expression of already acquired CRs. As illustrated in Figure $8 C$, the learning curve after the six lidocaine sessions seemed to start from baseline values suggesting the absence of nonexpressed CRs generated in circuits presynaptic to the IP nucleus. In fact, the percentage of CRs collected during the seventh and eighth sessions from the control group were significantly $\left(F_{(9,18)}=268.9 ; p<0.05\right)$ larger than those reached by the experimental group.

To sum up, lidocaine infusion into the IP nucleus greatly affected the expression of both conditioned and unconditioned eyelid responses for the whole training. The initial inactivation of the IP nucleus evoked a large increase in the spontaneous firing of $\mathrm{RN}$ neurons, an effect that decreased, but was still present during successive local injections of lidocaine. Nevertheless, these changes in firing rate could not be related to the acquisition process (see below; Fig. $8 B, C$ ).

The decrease in the amplitude of unconditioned responses following lidocaine or muscimol injections in the IP nucleus deserved a further comment. Indeed, some reported effects in rabbits are different from those reported here in the same species (Poulos et al., 2009). Nevertheless, we have collected similar findings in cats (Jiménez-Díaz et al., 2004) during classical conditioning of eyelid responses. In addition, there are early reports in rabbits indicating a decrease in the amplitude of unconditioned responses following the lesion and/or the inactivation of the IP nucleus (Welsh and Harvey, 1989; Chapman et al., 1990; Welsh, 1992; Wikgren and Korhonen, 2001). It has also been shown that the activation of IP neurons with GABA antagonists also facilitates unconditioned responses (Chen and Evinger, 2006). A possible explanation for this discrepancy is that most lesion studies performed in rabbits refer to a rostral location of IP neurons related to eyelid responses, a fact not confirmed in many other

\section{$\leftarrow$}

(Figure legend continued.) (left set of records) or with lidocaine (right set of records) in the MC during the six sessions. Note the decrease in EMG area corresponding to conditioned and unconditioned responses following lidocaine administration. $\boldsymbol{D}$, From top to bottom are illustrated the evolution of (Rs (\%), EMG area (\% of change), and mean firing rate (spikes/s) for the experiment illustrated in $C(n=3$ animals/group). Note the decrease in the percentage of CRs, EMG area, and neuronal firing rate following lidocaine injection in the $M C\left({ }^{*} p \leq 0.05\right.$, two-way ANOVA). species (mice, rats, cats, monkeys; Sánchez-Campusano et al., 2011). In all of these species eyelid-related neurons are located in more caudal aspects of the IP nucleus as performed here. This anatomical discrepancy could explain the different effects produced by lidocaine injections in deep cerebellar nuclei. In addition, bolus injection of lidocaine has a rather transient effect compared with the continuous perfusion carried put here (see Discussion).

\section{Comparative analysis of the effects of lidocaine injection in the MC and in the IP nucleus on increases in firing rates of RN neurons during the CS-US interval}

The inactivation of the cerebellar IP nucleus (Fig. 7D, bottom graph) produced an initial activation of the spontaneous firing of $\mathrm{RN}$ and pararubral type A and B neurons less noticeable than during MC inactivation (Fig. $6 \mathrm{D}$, bottom graph). Since this activation was rather unspecific and not related to the learning situation (Fig. $7 B$ ), we performed a further analysis of unitary data collected during the CS-US interval. In this case, and to consider exclusively the increase in firing rate evoked by the paired CS-US presentation, we removed the spontaneous firing presented by the recorded neuron (Fig. $8 \mathrm{~A}$ ). For this, we subtracted from the firing rate computed during the CS-US interval the value of the mean firing rate collected immediately $(250 \mathrm{~ms})$ before CS presentation. In this situation, values from control animals (Fig. $8 \mathrm{~B}$ ) presented an inverted- $U$ profile similar to the values already illustrated in the bottom graph of Figure $5 \mathrm{~A}$. In contrast, firing rates collected from $\mathrm{RN}$ neurons in animals injected with lidocaine in the MC best fit a linear regression line, with a positive slope ( $y=10.46+1.06 x ; r=0.88 ; p=0.022$; Fig. $8 B$ ), while firing rates collected from neurons recorded during lidocaine injections in the IP nucleus better fitted a decreasing linear regression line $(y=11.48-1.09 x ; r=0.9 ; p=0.015$; Fig. $8 B)$. These results further confirm the proposal of an active involvement of RN neurons in the learning process following the inactivation of the MC but not of the cerebellar IP nucleus.

\section{Comparative analysis of the effects of lidocaine injection in the MC and in the IP nucleus on unconditioned eyelid responses}

As shown in Figures $6 C$ and $7 C$, the performance of unconditioned responses was differentially affected by the inactivation of the $\mathrm{MC}$ and the cerebellar IP nucleus. Indeed, the inactivation of the MC evoked only a minor decrease in the performance of unconditioned responses (Fig. 6C), while the inactivation of the IP nucleus almost canceled out its expression (Fig. 7C). Figure 9 illustrates the evolution of unconditioned responses across the successive conditioning sessions. For this, we quantified the rectified EMG area (in $\mathrm{mV} \times \mathrm{s}$ ) of the $\mathrm{OO}$ activation evoked by the US (Fig. 9A). As seen in Figure 9B, the rectified EMG area in control animals was significantly larger $(p<0.001)$ than in animals injected with lidocaine in the MC and in the cerebellar IP nucleus. In addition, unconditioned eyelid responses evoked in MC animals injected with lidocaine were significantly larger $(p<$ 0.05 ) than values collected from animals injected with lidocaine in the IP nucleus. In accordance, the inactivation of cerebellar output has more-deleterious effects on corneal reflex circuits than does MC inactivation.

\section{Discussion}

\section{Principal findings of the present study}

We have reported here the firing activities of antidromically identified RN and pararubral neurons related to conditioned and 

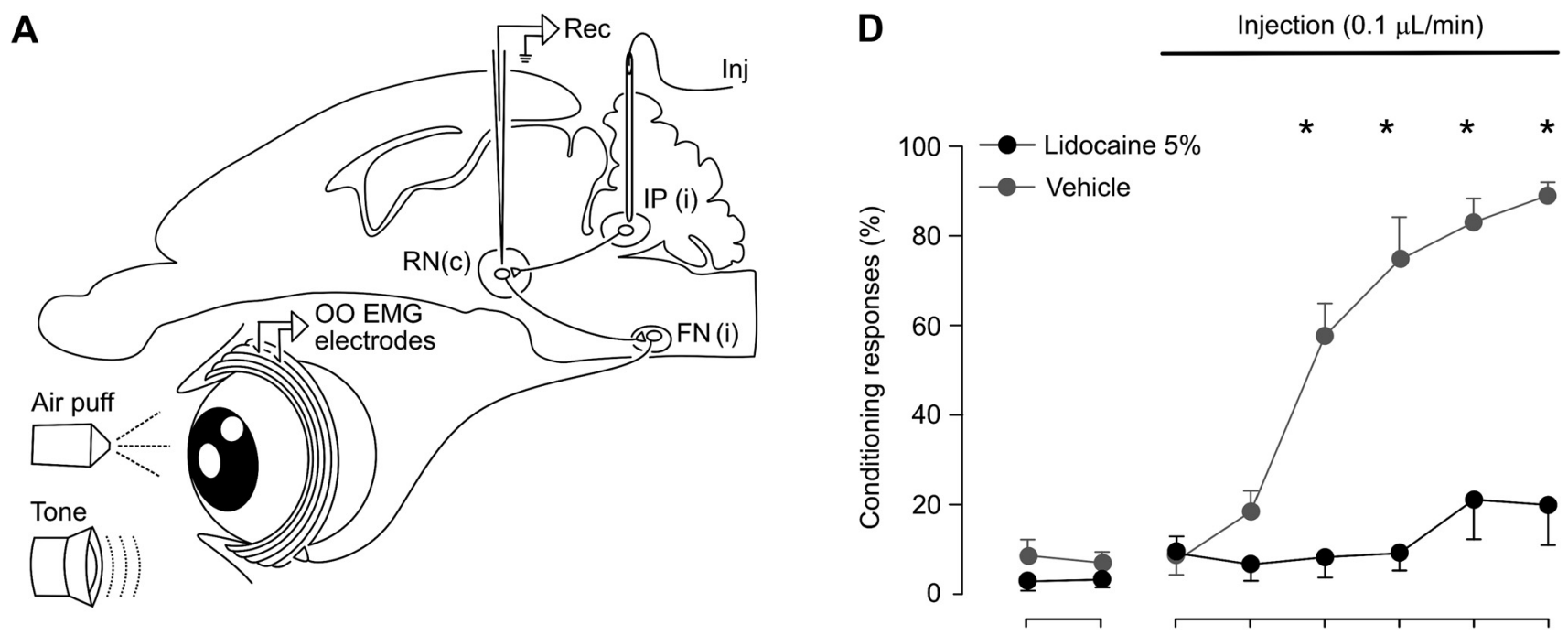

B

Injection $(0.1 \mu \mathrm{L} / \mathrm{min})$
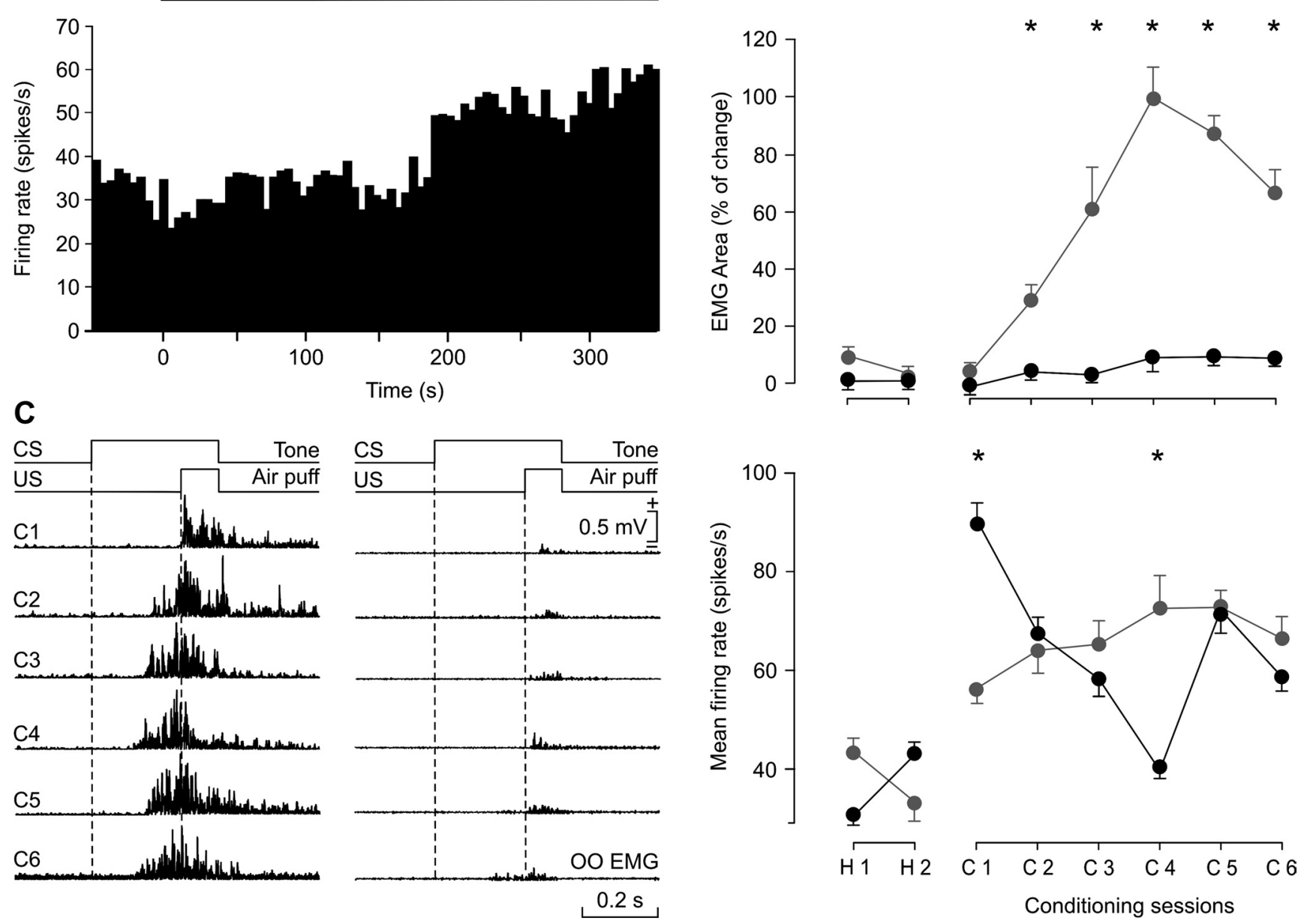

Figure 7. Effects of IP nucleus inactivation on learning curves and on the firing rate of RN neurons. $A$, Experimental design. The contralateral cerebellar IP nucleus was perfused with lidocaine ( $5 \%$ solution at a rate of $0.1 \mu \mathrm{l} / \mathrm{min}$ ) or vehicle (Inj) during classical eyeblink conditioning and unitary recordings (Rec) of RN neurons. $\boldsymbol{B}$, Increase in the spontaneous firing of identified RN neurons following lidocaine injection in the IP nucleus. C, Evolution of the rectified EMG activity of the 00 muscle across conditioning sessions in representative animals injected with vehicle (left set of records) or with lidocaine (right set of records) in the IP nucleus. Note the decrease in EMG area corresponding to conditioned and unconditioned responses following lidocaine administration. D, From top to bottom are illustrated the evolution of (Rs (\%), EMG area (\% of change), and mean firing rate (spikes/s) for the experiment illustrated in $C\left(n=3\right.$ animals/group). Note the decrease in the percentage of CRs and EMG area, and the changes in neuronal firing rate following lidocaine injection in the IP nucleus $\left({ }^{*} p \leq 0.01\right.$, two-way ANOVA). 


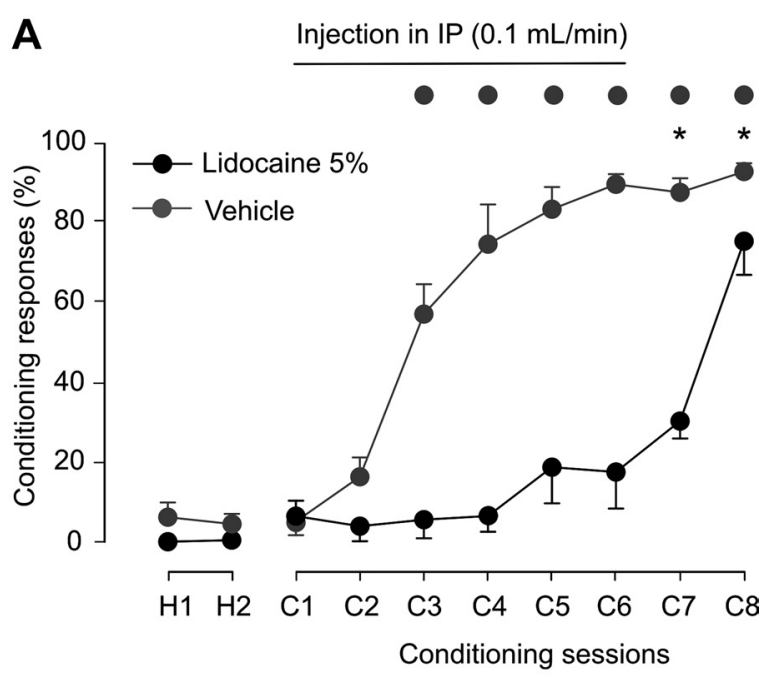

B
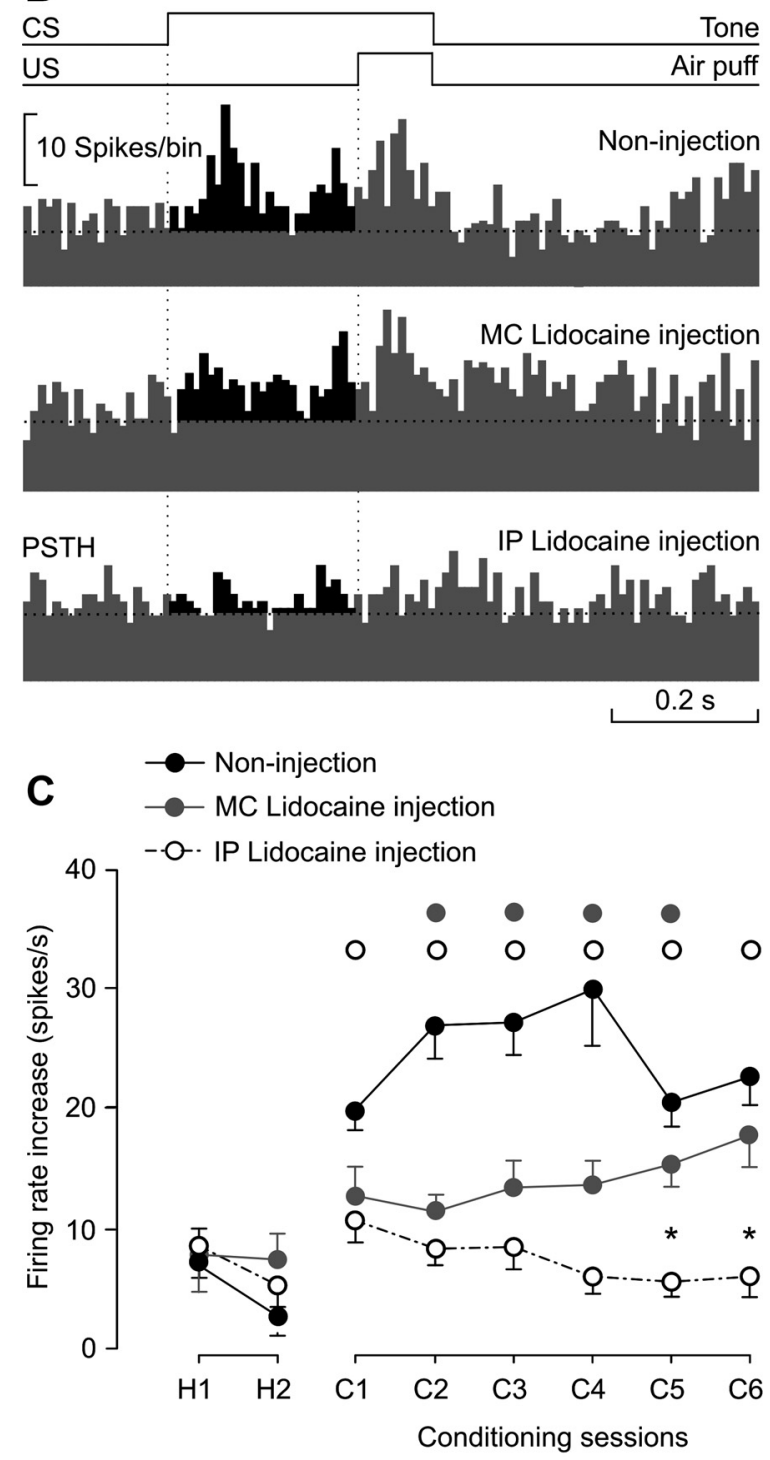

Figure 8. Quantitative analysis of changes evoked, during the CS-US interval, in the firing rate of identified RN neurons by lidocaine injections in the $M C$ or in the cerebellar IP nucleus. The recovery of CRs after lidocaine injections in the IP nucleus is also illustrated. $\boldsymbol{A}$, Effects of lidocaine injection in the IP during the first six conditioning sessions (top bar) on the acquisition of CRs ( $n=3$; black circles and lines) compared with control (vehicle-injected animals, $n=3$; gray
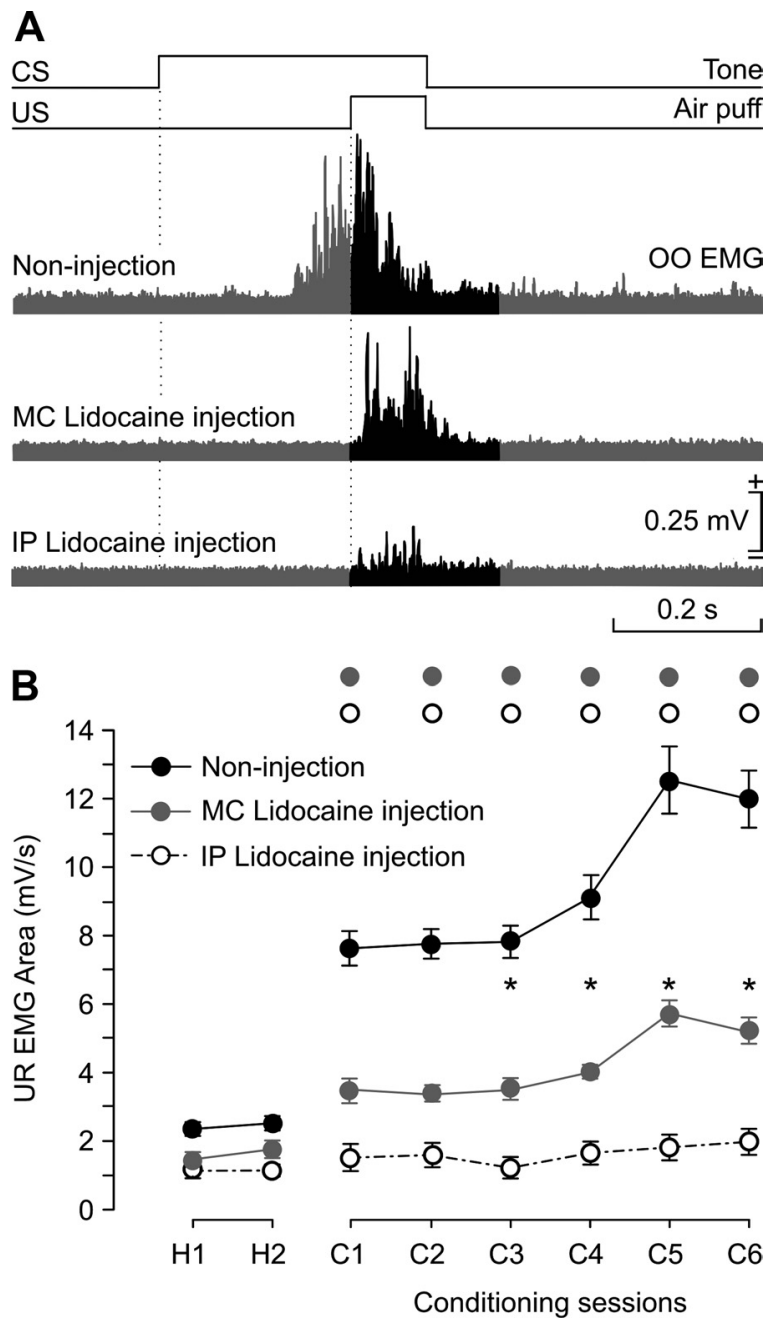

Figure 9. Quantitative analysis of changes evoked in unconditioned eyelid responses by lidocaine injections in the MC or in the cerebellar IP nucleus. $\boldsymbol{A}$, From top to bottom are illustrated the conditioning paradigm and rectified EMG profiles of the 00 muscle collected from representative noninjected, and MC- and IP-injected rabbits. Illustrated recordings were collected from the third conditioning session. In black is indicated the EMG area corresponding to the unconditioned response. $\boldsymbol{B}$, Evolution of unconditioned response EMG areas (in $\mathrm{mV} \times \mathrm{s}$ ) collected from noninjected (black circles and continuous line), and MC-injected (gray circles and lines) and IP-injected (white circles and dashed line) rabbits ( $n=3$ per group) during habituation and conditioning sessions. Gray and white circles at the top indicate significant ( $p \leq 0.01$, two-way ANOVA) differences of the noninjected group with MC- and IP-injected rabbits. ${ }^{*} p \leq 0.05$ for differences between the two injected groups.

circles and lines) values. Note the slow increase in the percentage of CRs after the end of lidocaine injections. Gray dots and asterisks at the top indicate significant ( $p \leq 0.05$, two-way ANOVA) differences with habituation values for the control and lidocaine-injected groups. $\boldsymbol{B}$, From top to bottom are illustrated the conditioning paradigm and the PSTH of representative RN neurons collected from noninjected, and MC- and IP-injected rabbits. Illustrated recordings were collected from the third conditioning session. In black are indicated the time windows (CS-US interval) from which the corresponding increases in firing rate were computed. C, Evolution of the increase in mean firing rates during the CS-US interval collected from noninjected (black circles and continuous line), and MC-injected (gray circles and lines) and IPinjected (white circles and dashed line) rabbits ( $n=3$ per group) during habituation and conditioning sessions. Gray and white circles at the top indicate significant ( $p \leq 0.01$, two-way ANOVA) differences of the noninjected group with MC- and IP-injected rabbits, and asterisks indicate significant ( $p \leq 0.05$, two-way ANOVA) differences observed between the two injected groups. 
unconditioned eyelid responses. These neurons seemed to project to the contralateral facial and/or the accessory abducens nuclei and were activated synaptically from the ipsilateral MC and from the contralateral IP nucleus. Interestingly, stimulation of the MC evoked long-latency responses, indicating the presence of local intrinsic circuits. At the same time, $\mathrm{RN}$ and pararubral neurons were activated by stimulations of different sensory modalities, suggesting that they could be capable of integrating sensory information with motor commands. Firing rates of these RN and pararubral neurons, during the CS-US interval, seemed to correlate better with the EMG activity of the OO muscle than with the learning curve, suggesting that they were more directly involved in the performance of CRs than in the acquisition process (Desmond and Moore, 1991; Porras-García et al., 2010). The reversible inactivation of the MC during conditioning sessions evoked a significant and lasting reduction in the percentage of CRs and in the firing rates of recorded neurons, with a minor effect on unconditioned responses. However, the firing rate of RN neurons started to recover from the fourth session on, in parallel with the increase in CRs and in the amplitude of the rectified EMG activity of the OO muscle. In contrast, inactivation of the IP nucleus evoked the almost complete disappearance of both conditioned and unconditioned responses for the six conditioning sessions, with a rebound increase of the spontaneous firing of RN neurons. As illustrated in Figure 8, this increase in firing rate of RN neurons during IP inactivation was not related to the learning process, while, as is also illustrated in Figure 8, the recovery of CRs and muscle activity during $\mathrm{MC}$ inactivation was probably due to the specific increase in firing rate of $\mathrm{RN}$ neurons during the CS-US interval. In general, the RN and its pararubral area seem capable of learning-dependent changes in synaptic strength, which become particularly evident in the absence of MC outputs.

\section{Different roles ascribed to red nucleus neurons}

The presence of a definite $\mathrm{RN}$ and of a rubrospinal tract seems to be related to the presence of limbs or limb-like structures, as in the case of many terrestrial vertebrates and of certain rays, respectively (ten Donkelaar, 1988). In relation to this widely accepted evolutionary trend, $\mathrm{RN}$ axonal projections to facial and accessory abducens nuclei- containing motoneurons innervating $\mathrm{OO}$ and eye retractor bulbi muscles, respectively - have usually been considered to be collaterals of magnocellular RN neurons projecting to the spinal cord (Courville, 1966; Mizuno and Nakamura, 1971; Hinrichsen and Watson, 1983). However, recent experiments performed with retrograde tracers and/or using rabies virus immunolabeling have convincingly shown that $\mathrm{RN}$ neurons projecting to the FN belong to a specific population located in the parvocellular part of the $\mathrm{RN}$ and occupying a dorsolateral position, as well as extending to the dorsally adjoining pararubral area (Ruigrok and Cella, 1995; Morcuende et al., 2002; Pong et al., 2008). As shown here, the stereotaxic reconstruction of recorded neurons belonging to types A and B presented a similar location within the nucleus. From a functional point of view, this finding makes sense, since facial motor functions cannot readily be associated with the control exerted by rubral neurons projecting to spinal cord motor centers related to limb displacements and/or to manipulative behaviors (Horn et al., 2002; Miller and Gibson, 2009).

With respect to the classical conditioning of eyelid responses, the $\mathrm{RN}$ has usually been considered an important component of the circuit originated in the cerebellar cortex and/or IP nucleus, having in this regard a mere relay and premotor function. In a seminal study, Chapman et al. (1990) performed multiunitary recordings of putative $\mathrm{RN}$ neurons and the reversible lidocaine inactivation of both red and IP nuclei during classical conditioning of behaving rabbits, concluding that the $\mathrm{RN}$ acts as a relay nucleus for motor commands generated at cerebellar circuits. In this regard, it has been reported that RN inactivation produced no effect on the acquisition process (Grant and HorcholleBossavit, 1986; Clark and Lavond, 1993; Krupa et al., 1993; Robleto and Thompson, 2008), a point of view still accepted by many groups (Bracha et al., 2009; Freeman and Steinmetz, 2011). In dissension, early (Kennedy and Humphrey, 1987; Schmied et al., 1988; Cartford et al., 1997) and recent studies (Miller and Gibson, 2009) proposed that the RN plays a more active role in motor learning processes. According to the present results, the RN seems to occupy a strategic position, receiving descending, and different, motor control commands from the cerebral and cerebellar circuits, which regulate its functioning. However, the putative active role in the acquisition of new motor abilities is noticed only when those afferent inputs are transiently removed during the acquisition process. In this regard, it is very important to mention that lidocaine inactivation has a short-lasting $(\approx 20$ min) effect when injected in a single bolus (Martin and Ghez, 1999). For this very reason, we decided to inject lidocaine in a continuous manner during the whole conditioning session. This precise experimental approach could explain the different results reported here with regard to the inactivation of both MC and IP nucleus.

\section{Learning-dependent changes in the neuronal activity of RN and pararubral neurons during classical eyeblink conditioning}

In clear opposition to the proposal that the $\mathrm{RN}$ is a mere relay premotor center conveying motor commands emanating from the cerebellum - the latter being the proper neuronal site where the acquisition and storage of newly acquired eyelid conditioned responses take place (Chapman et al., 1990; Bracha et al., 1993, 2009; Krupa et al., 1993; Freeman and Steinmetz, 2011)—the RN was one of the first places where activity-dependent changes in synaptic strength was supposed to occur (Tsukahara, 1981). In a series of seminal experiments, Tsukahara et al. (1981) and Murakami et al. (1987, 1988), as well as additional groups (Ito and Oda, 1994; Pananceau et al., 1996), suggested that RN magnocellular neurons could be capable of synaptic plastic changes during the acquisition of different types of skeletal associative learning tasks. These changes in synaptic strength could take place at the distal dendrites of magnocellular neurons, the preferential place of innervation of cortical sensorimotor neurons projecting to the RN (Tsukahara et al., 1967; Pizzini et al., 1975; Eccles, 1986). The present results reopen the possibility of the presence of learningdependent mechanisms present in RN and pararubral neurons, which can easily be noticed in the absence of the proper activity of MC circuits, but not after the reversible inactivation of cerebellar outputs, and merit a detailed account.

There are early reports indicating that rabbits are capable of acquiring classically conditioned nictitating membrane responses in decerebrate-decerebellate conditions (Kelly et al., 1990), suggesting the presence of additional neural centers with a (limited) capacity for associative learning. More recently, it has been shown in behaving mice that the occlusion of hippocampal circuits by the experimental induction of long-term potentiation still allows the expression of a low rate $(<40 \%)$ of eyelid CRs (Gruart et al., 2006). Indeed, the RN and the pararubral area are suitable for this role, because, in addition to the considerable inputs reaching it from cerebral cortex and cerebellar pathways, 
they also receive afferent activation from different sensory modalities (Padel et al., 1988) and, as recently shown, there are intrinsic circuits and synaptic activations within the RN and the pararubral area (Horn et al., 2002), including GABAergic and non-GABAergic neurons (Haley et al., 1988). The different effects produced on classical eyeblink conditioning by the transient inactivation of the cerebral cortex and the cerebellar IP nucleus could be explained by their different innervation of RN neurons. Cerebral cortical axonal projections seem to synapse on distal dendrites activating NMDA and non-NMDA glutamate receptors (Davies et al., 1994), while IP axons seem to terminate on non-NMDA receptors located preferentially on the soma and proximal dendrites of rubral neurons (Billard et al., 1991). Thus, and as originally proposed by Tsukahara et al. (1981), the presence of NMDA receptors could explain the delayed changes in synaptic strength taking place in rubral circuits after the inactivation of the sensorimotor cortex. It is still possible that the compensating learning mechanisms observed in rubral neurons after cerebral cortex inactivation could be the result of a delayed activation of the rubro-olivary-cerebellar circuit (Kennedy and Humphrey, 1987). However, the problem with proposing the rubro-olivary-cerebellar pathway as the one involved in the acquisition process is that this pathway seems to have no ready access to the NMDA receptors located on the membrane of rubral neurons, and that IP nucleus effects on the acquisition process seem to affect conditioned and unconditioned responses equally, their being related more with CR performance, and not with the acquisition process. In fact, and as shown here, the loss of IP neuron responses prevents the proper activity of rubral and pararubral neurons (Figs. 7, 8) and its precise contribution to the acquisition process.

In relation with the above contentions, the $\mathrm{RN}$ could have a role in the appropriated timing of CRs. These timing activities could be generated by feedback circuits involving RN, cerebellar IP, and sensory inputs (Houk, 1991; Cartford et al., 1997). The acute disappearance of facilitatory inputs from IP neurons could block these reverberant activities and prevent the learningdepending evolution of rubral activities related to the generation and performance of eyelid CRs. In addition, a reinforcing role of the IP nucleus for the expression of both conditioned and unconditioned eyelid responses has been already shown in recording and/or transient inactivation studies performed in behaving cats (Jiménez-Díaz et al., 2004) and mice (Porras-García et al., 2010). These two phenomena could explain why its inactivation has such a deleterious effects on both conditioned and unconditioned eyelid responses.

The disfacilitatory effects of IP inactivation cannot only be mediated by IP projections to the RN, but also by larger projections to motor and premotor cerebral cortical circuits. These functional changes could also help to explain why acute removal of cerebellar facilitatory outputs has such a deleterious effect on $\mathrm{RN}$ functions.

In contrast to the learning-dependent effects activated by the transient inactivation of the MC, the inactivation of the IP nucleus should produce a strong disfacilitation of $\mathrm{RN}$ and pararubral neuron firing, because of the strategic location of their axonal terminal boutons in somatic and proximal dendrite sites. The significant increase in the spontaneous firing of rubral neurons following IP nucleus inactivation can tentatively be explained by the disfacilitation of GABAergic neurons (Haley et al., 1988) present in RN intrinsic circuits. In this regard, Nieoullon et al. (1988) proposed that the inactivation of the cerebellum could be compensated by an unspecific increase of corticorubral pro- jections together with a decrease in the activity of rubral GABAergic interneurons. These two separate activities could help to explain the increase in basal activity observed in $\mathrm{RN}$ neurons during the inactivation of the IP nucleus.

\section{References}

Billard JM, Daniel H, Pumain R (1991) Sensitivity of rubrospinal neurons to excitatory amino acids in the rat red nucleus in vivo. Neurosci Lett 134:49-52.

Bracha V, Stewart SL, Bloedel JR (1993) The temporary inactivation of the red nucleus affects performance of both conditioned and unconditioned nictitating membrane responses in the rabbit. Exp Brain Res 94:225-236.

Bracha V, Zbarska S, Parker K, Carrel A, Zenitsky G, Bloedel JR (2009) The cerebellum and eye-blink conditioning: learning versus network performance hypotheses. Neuroscience 162:787-796.

Canedo A, Lamas JA (1989) Rubrospinal tract of the cat: superposition of antidromic responses and changes in axonal excitability following orthodromic activity. Brain Res 502:28-38.

Cartford MC, Gohl EB, Singson M, Lavond DG (1997) The effects of reversible inactivation of the red nucleus on learning-related and auditoryevoked unit activity in the pontine nuclei of classically conditioned rabbits. Learn Mem 3:519-531.

Case GR, Lavond DG, Thompson RF (2002) Cortical spreading depression and involvement of the motor cortex, auditory cortex, and cerebellum in eyeblink classical conditioning of the rabbit. Neurobiol Learn Mem 78:234-245.

Chapman PF, Steinmetz JE, Sears LL, Thompson RF (1990) Effects of lidocaine injection in the interpositus nucleus and red nucleus on conditioned behavioral and neuronal responses. Brain Res 537:149-156.

Chen FP, Evinger C (2006) Cerebellar modulation of trigeminal reflex blinks: interpositus neurons. J Neurosci 26:10569-10576.

Clark RE, Lavond DG (1993) Reversible lesions of the red nucleus during acquisition and retention of a classically conditioned behavior in rabbits. Behav Neurosci 107:264-270.

Courville J (1966) Rubrobulbar fibres to the facial nucleus and the lateral reticular nucleus (nucleus of the lateral funiculus). An experimental study in the cat with silver impregnation methods. Brain Res 1:317-337.

Davies J, Qume M, Harris NC (1994) Pharmacological characterisation of excitatory synaptic transmission in the cat red nucleus in vivo. Brain Res 649:43-52.

Desmond JE, Moore JW (1991) Single-unit activity in red nucleus during the classically conditioned rabbit nictitating membrane response. Neurosci Res 10:260-279.

Eccles JC (1986) The investigations of N. Tsukahara on relocation of synapses on red nucleus neurones. Neurosci Res 3:476-486.

Eccles JC, Scheid P, Táboríková H (1975) Responses of red nucleus neurons to antidromic and synaptic activation. J Neurophysiol 38:947-964.

Freeman JH, Steinmetz AB (2011) Neural circuitry and plasticity mechanisms underlying delay eyeblink conditioning. Learn Mem 18:666-677.

Girgis M, Shih-Chang W (1981) A new stereotaxic atlas of the rabbit brain. St. Louis: Warren H. Green.

Godefroy JN, Thiesson D, Pollin B, Rokyta R, Azerad J (1998) Reciprocal connections between the red nucleus and the trigeminal nuclei: a retrograde and anterograde tracing study. Physiol Res 47:489-500.

Grant K, Horcholle-Bossavit G (1986) Red nucleus inputs to retractor bulbi motoneurones in the cat. J Physiol 371:317-327.

Gruart A, Schreurs BG, del Toro ED, Delgado-García JM (2000) Kinetic and frequency-domain properties of reflex and conditioned eyelid responses in the rabbit. J Neurophysiol 83:836-852.

Gruart A, Streppel M, Guntinas-Lichius O, Angelov DN, Neiss WF, DelgadoGarcía JM (2003) Motoneuron adaptability to new motor tasks following two types of facial-facial anastomosis in cats. Brain 126:115-133.

Gruart A, Muñoz MD, Delgado-García JM (2006) Involvement of the CA3$\mathrm{CAl}$ synapse in the acquisition of associative learning in behaving mice. J Neurosci 26:1077-1087.

Gruber P, Gould DJ (2010) The red nucleus: past, present, and future. Neuroanatomy 9:1-3.

Haley DA, Thompson RF, Madden J 4th (1988) Pharmacological analysis of the magnocellular red nucleus during classical conditioning of the rabbit nictitating membrane response. Brain Res 454:131-139.

Hinrichsen CF, Watson CD (1983) Brain stem projections to the facial nucleus of the rat. Brain Behav Evol 22:153-163. 
Horn KM, Pong M, Batni SR, Levy SM, Gibson AR (2002) Functional specialization within the cat red nucleus. J Neurophysiol 87:469-477.

Houk JC (1991) Red nucleus: role in motor control. Curr Opin Neurobiol 1:610-615.

Huisman AM, Kuypers HG, Verburgh CA (1981) Quantitative differences in collateralization of the descending spinal pathways from red nucleus and other brain stem cell groups in rat as demonstrated with the multiple fluorescent retrograde tracer technique. Brain Res 209:271-286.

Huisman AM, Kuypers HG, Verburgh CA (1982) Differences in collateralization of the descending spinal pathways from red nucleus and other brain stem cell groups in cat and monkey. Prog Brain Res 57:185-217.

Ito M, Oda Y (1994) Electrophysiological evidence for formation of new corticorubral synapses associated with classical conditioning in the cat. Exp Brain Res 99:277-288.

Ivkovich D, Thompson RF (1997) Motor cortex lesions do not affect learning or performance of the eyeblink response in rabbits. Behav Neurosci 111:727-738.

Jiménez-Díaz L, Navarro-López Jde D, Gruart A, Delgado-García JM (2004) Role of cerebellar interpositus nucleus in the genesis and control of reflex and conditioned eyelid responses. J Neurosci 24:9138-9145.

Kelly TM, Zuo CC, Bloedel JR (1990) Classical conditioning of the eyeblink reflex in the decerebrate-decerebellate rabbit. Behav Brain Res 38:7-18.

Kennedy PR, Humphrey DR (1987) The compensatory role of the parvocellular division of the red nucleus in operantly conditioned rats. Neurosci Res 5:39-62.

Kotani S, Kawahara S, Kirino Y (2002) Classical eyeblink conditioning in decerebrate guinea pigs. Eur J Neurosci 15:1267-1270.

Krupa DJ, Thompson JK, Thompson RF (1993) Localization of a memory trace in the mammalian brain. Science 260:989-991.

Leal-Campanario R, Fairén A, Delgado-García JM, Gruart A (2007) Electrical stimulation of the rostral medial prefrontal cortex in rabbits inhibits the expression of conditioned eyelid responses but not their acquisition. Proc Natl Acad Sci U S A 104:11459-11464.

Martin JH, Ghez C (1999) Phamacological inactivation in the analysis of the central control of movement. J Neurosci Methods 86:145-159.

Megirian D, Bures J (1970) Unilateral cortical spreading depression and conditioned eyeblink responses in the rabbit. Exp Neurol 27:34-45.

Mendez I, Hong M (1997) Reconstruction of the striato-nigro-striatal circuitry by simultaneous double dopaminergic grafts: a tracer study using fluorogold and horseradish peroxidase. Brain Res 778:194-205.

Miller LE, Gibson AR (2009) Red nucleus. In: Encyclopedia of neuroscience, Vol 8 (Squire LR ed), pp 55-62. Oxford: Academic.

Mizuno N, Nakamura Y (1971) Rubral fibers to the facial nucleus in the rabbit. Brain Res 28:545-549.

Morcuende S, Delgado-García JM, Ugolini G (2002) Neuronal premotor networks involved in eyelid responses: retrograde transneuronal tracing with rabies virus from the orbicularis oculi muscle in the rat. J Neurosci 22:8808-8818.

Múnera A, Gruart A, Muñoz MD, Fernández-Mas R, Delgado-García JM (2001) Hippocampal pyramidal cell activity encodes conditioned stimulus predictive value during classical conditioning in alert cats. J Neurophysiol 86:2571-2582.

Murakami F, Higashi S, Katsumaru H, Oda Y (1987) Formation of new corticorubral synapses as a mechanism for classical conditioning in the cat. Brain Res 437:379-382.

Murakami F, Oda Y, Tsukahara N (1988) Synaptic plasticity in the red nucleus and learning. Behav Brain Res 28:175-179.

Nieoullon A, Vuillon-Cacciuttolo G, Dusticier N, Kerkérian L, André D, Bosler O (1988) Putative neurotransmitters in the red nucleus and their involvement in postlesion adaptive mechanisms. Behav Brain Res 28:163-174

Oakley DA, Russell IS (1972) Neocortical lesions and Pavlovian conditioning. Physiol Behav 8:915-926.

Padel Y, Sybirska E, Bourbonnais D, Vinay L (1988) Electrophysiological identification of a somaesthetic pathway to the red nucleus. Behav Brain Res 28:139-151.

Pananceau M, Rispal-Padel L, Meftah EM (1996) Synaptic plasticity of the interpositorubral pathway functionally related to forelimb flexion movements. J Neurophysiol 75:2542-2561.

Pauletti G, Berardelli A, Cruccu G, Agostino R, Manfredi M (1993) Blink reflex and the masseter inhibitory reflex in patients with dystonia. Mov Disord 8:495-500.

Pizzini G, Tredici G, Miani A (1975) Corticorubral projection in the cat. An experimental electronmicroscopic study. J Submicr Cytol 7:231-238.

Pong M, Horn KM, Gibson AR (2008) Pathways for control of face and neck musculature by the basal ganglia and cerebellum. Brain Res Rev 58:249-264.

Porras-García E, Sánchez-Campusano R, Martínez-Vargas D, Domínguezdel-Toro E, Cendelín J, Vozeh F, Delgado-García JM (2010) Behavioral characteristics, associative learning capabilities, and dynamic association mapping in an animal model of cerebellar degeneration. J Neurophysiol 104:346-365.

Poulos AM, Nobuta H, Thompson RF (2009) Disruption of cerebellar cortical inhibition in the absence of learning promotes sensory-evoked eyeblink responses. Behav Neurosci 123:694-700.

Rieke F, Warland D, van Steveninck RdR, Bialek W (1997) Spikes. Exploring the neural code. Cambridge, MA: MIT.

Robinson FR, Rice PM, Holleman JR, Berger TW (2001) Projection of the magnocellular red nucleus to the region of the accessory abducens nucleus in the rabbit. Neurobiol Learn Mem 76:358-374.

Robleto K, Thompson RF (2008) Extinction of a classically conditioned response: red nucleus and interpositus. J Neurosci 28:2651-2658.

Ruigrok TJH, Cella F (1995) Precerebellar nuclei and red nucleus. In: The rat nervous system (Paxinos G, ed), pp 277-308. San Diego: Academic.

Sánchez-Campusano R, Gruart A, Delgado-García JM (2011) Dynamic changes in the cerebellar-interpositus/red-nucleus-motoneuron pathway during motor learning. Cerebellum 10:702-710.

Schmied A, Amalric M, Dormont JF, Condé H, Farin D (1988) Participation of the red nucleus in motor initiation: unit recording and cooling in cats. Behav Brain Res 28:207-216.

ten Donkelaar HJ (1988) Evolution of the red nucleus and rubrospinal tract. Behav Brain Res 28:9-20.

Tsukahara N (1981) Synaptic plasticity in the mammalian central nervous system. Annu Rev Neurosci 4:351-379.

Tsukahara N, Toyama K, Kosaka K (1967) Electrical activity of red nucleus neurones investigated with intracellular microelectrodes. Exp Brain Res 4:18-33.

Tsukahara N, Oda Y, Notsu T (1981) Classical conditioning mediated by the red nucleus in the cat. J Neurosci 1:72-79.

Welsh JP (1992) Changes in the motor pattern of learned and unlearned responses following cerebellar lesions: a kinematic analysis of the nictitating membrane reflex. Neuroscience 47:1-19.

Welsh JP, Harvey JA (1989) Cerebellar lesions and the nictitating membrane reflex: performance deficits of the conditioned and unconditioned response. J Neurosci 9:299-311.

Wikgren J, Korhonen T (2001) Interpositus nucleus inactivation reduces unconditioned response amplitude after paired but not explicitly unpaired treatment in rabbit eyeblink conditioning. Neurosci Lett 308:181184. 